\title{
REVIEW
}

\section{Parental investment, sexual selection and sex ratios}

\author{
HANNA KOKKO* \& MICHAEL D. JENNIONS ${ }^{\dagger}$ \\ "Laboratory of Ecological and Evolutionary Dynamics, Department of Biological and Environmental Science, University of Helsinki, Helsinki, Finland \\ 'School of Botany $\otimes$ Zoology, The Australian National University, Canberra, ACT, Australia
}

\section{Keywords:}

mating success;

parental care;

parental investment;

sex ratio;

sex roles;

sexual selection.

\begin{abstract}
Conventional sex roles imply caring females and competitive males. The evolution of sex role divergence is widely attributed to anisogamy initiating a self-reinforcing process. The initial asymmetry in pre-mating parental investment (eggs vs. sperm) is assumed to promote even greater divergence in postmating parental investment (parental care). But do we really understand the process? Trivers [Sexual Selection and the Descent of Man 1871-1971 (1972), Aldine Press, Chicago] introduced two arguments with a female and male perspective on whether to care for offspring that try to link pre-mating and post-mating investment. Here we review their merits and subsequent theoretical developments. The first argument is that females are more committed than males to providing care because they stand to lose a greater initial investment. This, however, commits the 'Concorde Fallacy' as optimal decisions should depend on future pay-offs not past costs. Although the argument can be rephrased in terms of residual reproductive value when past investment affects future pay-offs, it remains weak. The factors likely to change future pay-offs seem to work against females providing more care than males. The second argument takes the reasonable premise that anisogamy produces a male-biased operational sex ratio (OSR) leading to males competing for mates. Male care is then predicted to be less likely to evolve as it consumes resources that could otherwise be used to increase competitiveness. However, given each offspring has precisely two genetic parents (the Fisher condition), a biased OSR generates frequencydependent selection, analogous to Fisherian sex ratio selection, that favours increased parental investment by whichever sex faces more intense competition. Sex role divergence is therefore still an evolutionary conundrum. Here we review some possible solutions. Factors that promote conventional sex roles are sexual selection on males (but non-random variance in male mating success must be high to override the Fisher condition), loss of paternity because of female multiple mating or group spawning and patterns of mortality that generate female-biased adult sex ratios (ASR). We present an integrative model that shows how these factors interact to generate sex roles. We emphasize the need to distinguish between the ASR and the operational sex ratio (OSR). If mortality is higher when caring than competing this diminishes the likelihood of sex role divergence because this strongly limits the mating success of the earlier deserting sex. We illustrate this in a model where a change in relative mortality rates while caring and competing generates a shift from a mammalian type breeding system (female-only care, male-biased OSR and female-biased ASR) to an avian type system (biparental care and a male-biased OSR and ASR).
\end{abstract}




\section{Introduction}

\subsection{The taxonomy of sex roles}

In species where parents provide post-mating parental care, how much each sex contributes varies among taxa. In mammals, male-only care is completely absent and females care alone in about $90 \%$ of species (CluttonBrock, 1991). In birds, 90\% of species show biparental care (this includes $9 \%$ of species where cooperative helpers assist, Cockburn, 2006). Bird species with biparental care vary in the relative investment each sex makes into the different stages of parental care. On average, however, females invest more heavily than males (e.g. Møller \& Birkhead, 1993; Schwagmeyer et al., 1999). In reptiles with parental care this is provided either by the female or by both parents (Reynolds et al., 2002). In amphibians, male-only and female-only care has evolved equally often, while biparental care occurs at a low level (Beck, 1998; Summers et al., 2006, 2007). In fish, the ratio of genera with male-only to biparental to female-only care is 9:3:1 (Reynolds et al., 2002), but which sex provides the bulk of care varies widely among families (e.g. Goodwin et al., 1998, 2002; Mank et al., 2005). Finally, in invertebrate species with parental care the majority have female-only care, biparental care is uncommon, and male-only care is rare (Zeh $\delta$ Smith, 1985). In arthropods, for example, male care has only evolved in eight independent lineages (Tallamy, 2000).

This taxonomic list makes it clear that care patterns vary widely. In general, however, female care is more widespread than male care. With the notable exception of fish, this summary remains robust whether it is based on the number of independent evolutionary transitions from no care to caring by each sex, or on a simple tally of species (Kokko \& Jennions, 2003a, b). A similar taxonomic survey also reveals widespread variation in the level of male-male and female-female competition. However, observable mating competitiveness, personified in the development of weaponry, tends to be far more strongly developed in males than in females (Berglund et al., 1996). In general, males compete more intensely than females for matings.

Explaining taxonomic differences in sex biases in parental care and sexual competition will always involve historic contingencies. Specifically, unique features of a taxon's natural history must alter the trade-off between key fitness components in at least one sex. In fish, for example, male parental care appears to involve fewer opportunity costs (lost matings) than is the case in other taxa. This is because males who guard eggs can still attract new mates. Moreover, females sometimes prefer males that are already providing care (Reynolds \& Jones, 1999; Hale \& St Mary, 2007), and there is even recent evidence that male care can increase his certainty of paternity (Ah-King et al., 2005; Kvarnemo, 2006).

\subsection{How do sex roles evolve? Previous answers and an integrated modelling approach}

In this review we do not dwell on taxon-specific explanations because we want to focus on a more fundamental question: what factors create the asymmetry that biases females towards caring for offspring and males towards competing for mates? This asymmetry is the assumed default outcome of sex role evolution. It is reflected in the terminology of 'conventional' and 'reversed' sex roles. There is a perceived 'reversal' from normality whenever females compete for males and/or males provide more care than females (strictly speaking, 'sex roles' refer to the competition aspect but the focal point in this review is the frequent association with sexbiased care). Given an ancestral state where parental care is absent, is it correct to predict that competitive males and caring females evolve more easily than the reverse? More importantly, if so, why? In this review we discuss the insights and shortcomings of previous verbal arguments. We then build a formal mathematical model that integrates the main factors identified by others as important to assess how sex roles evolve given clearly stated assumptions.

To avoid a mystery novel format where we only reveal the answer in the last chapter, we will now briefly list the key arguments and assumptions that inform our mathematical model. Their importance will become apparent along the way (for an informal summary see Box 1). Our starting point is Trivers (1972) who first identified the intimate relationships between parental investment, the availability of mates and sexual selection. There are, however, several problems inherent in his subsequent verbal account of sex role evolution. First, there was a poorly substantiated claim that past investment is the main determinant of the current benefits that each sex can gain from providing additional parental care. We call this the 'female argument' and we explain why it largely fails to explain sex role divergence. Second, there was an implicit assumption that the best evolutionary response to competition is to invest more heavily into traits that increase competitiveness. Unfortunately this 'male argument' assumes the very fact it sets out to explain: namely, that slight asymmetries in competitive tendencies become exaggerated. Additionally, it conflicts with the observation that in many species males do invest heavily into traits, such as parental care or mate guarding, that decrease their ability to compete for new mates.

In amending both arguments we draw heavily on the fact that the options available to each sex must take into account the so-called Fisher condition (sensu Houston $\&$ McNamara, 2005): males cannot, on average, reproduce faster than females if the adult sex ratio (ASR) is even. This population-level constraint makes it incorrect to argue that males care less than females because they can compensate more rapidly by producing new offspring if the current ones die due to a lack of care. The 


\section{Box 1. A synopsis: two gobies chatting}

1. A: I've been listening to these researchers trying to explain how to get from anisogamy to conventional sex roles of competing males and caring females.

2. B: I heard them talk about the 'female' and the 'male' argument. 3. A: Yes, that's what they call two famous arguments that have been around for the last few decades. The female argument says that females can't afford to lose their past investment in offspring, which is typically larger than that of the male, so they care more for their kids.

4. B: Sounds logical, given that females lay large eggs and males make tiny sperm.

5. A: Yes, but back in the 1970s it was already pointed out that it's a

'Concorde fallacy' to keep investing simply because you've already invested lots.

6. B: Okay, females shouldn't make that daft mistake, but isn't it still valid to argue that if offspring die, females typically can't compensate as fast as males can?

7. A: No. Not if you believe people like Düsing, Fisher, or Queller. They say that in a population with equal numbers of males and females, males can't really produce offspring any faster than females. In their dreams males might be able to compensate, but in reality they're inescapably limited by female reproduction. They call it 'The Fisher Condition'.

8. B: But perhaps some males do better than others - doesn't that matter? 9. A: Yes, and these researchers I've been listening to promise to get back to that.

10. B: And what if there aren't equal numbers of males and females in the population?

11. A: Good point. They promise to get back to that too.

12. B: Wait, I just thought of something. I'm not sure the fallacy is a fallacy. What if past investment changes the parent's state? Can't this influence what each sex should do in future?

13. A: True, but if you look at how the states are likely to change, and assume that care-giving is costly, it's not so clear that the sex who has so far invested more and is now in poorer condition should keep investing. So as a general explanation the 'female argument' fails.

14. B: So what about the 'male argument' you mentioned earlier?

15. A: It says that when females care a bit more than males (by making eggs not sperm), there are more males searching for females than vice versa. This creates self-reinforcing selection, because males become mate-limited, have to spend lots in competitive traits, and don't have the time to care. So, however small the initial asymmetry in parental

investment, it tends to increase.

16. B: That sounds pretty good to me.

17. A: Until you think about all the other things a male could do. Like, care more. And sometimes they do. So do you really want to explain sex role divergence by telling males that if they're in a competitive situation, they can do nothing other than compete more?

18: B: I see, I slipped into assuming that from a slight asymmetry in how much a sex must compete we must end up with a stronger asymmetry. It sounded so logical but it doesn't really explain why the strengthening happens.

19: A: Yes, cheeky indeed, you just asserted that it must happen. But why should all males invest in competing when so few succeed there? They would do that if it was their only option, but we've both seen the world and we know that males often do other things. Seahorses are such dedicated fathers.

20: B: I agree. Males could respond to strong mating competition in other ways than investing in weaponry or being sexy. They could have bigger testes or mate guard more or, indeed, care more. If we want to explain parental investment from first principles, shouldn't we specify what alternatives males, as well as females, have?

21: A: Exactly. And these researchers point out that there can be an important time scale effect. In the short term competitiveness might change more quickly than care, but over evolutionary time, care patterns should respond too. So if competition to get new mates gets really tough, why not evolve to spend a bit more time with the current brood? 22. B: These time scale problems could make empirical tests really tricky. 23. A: Well, I think they like empiricists. They just want them to keep this in mind when interpreting results. And they also point out that theoreticians haven't always thought through these issues either.

24. B: But now l'm a bit confused. If the mate-limited sex finds it really hard to find new mates, and either sex can do something useful for offspring survival, then shouldn't it be especially beneficial for the mate-limited sex to care more?

25: A: Indeed. It is a bit like Fisherian sex ratio selection.

26: B: What do you mean?

27: A: It's always going to be hard to reproduce when you're a member of the overrepresented sex. An overrepresented sex (counted at birth) shouldn't be produced if parents can adjust offspring sex ratios. That's sex ratio theory for you. Now in the parental investment context, an overrepresented sex (counted in the mating pool) shouldn't be too keen to return to the mating pool if it's hard to succeed there, and if staying away to care for the kids brings about alternative benefits.

28: B: But that really sounds like the wrong prediction. You are telling me that sex roles can't diverge and will instead tend towards egalitarian care. But l've seen those hugely dimorphic elephant seals out there... and actually not that many species with egalitarian sex roles.

29: A: Yes, but it is nice to examine a factor that might prevent complete divergence. Without it, it would be really hard to explain why sex roles haven't always diverged to the extreme.

30. B: I sort of see that, but surely these researchers will have to come up with something that tends to pull the roles apart too?

31. A: Yes, and they discuss several options. One is multiple mating. If there is, for example, internal fertilization and females mate multiply, then a male parent is less certain about parentage than a female.

32. B: Yeh, that's a pretty common scenario.

33. A: Certainly. Another factor is that the Fisher condition doesn't mean some males' reproductive rates couldn't be closer to their dream potential than that of the average male in the population.

34. B: I think I already asked about that (point 8).

35. A: Yes you did. There are actually at least two different options here. It could be that a male knows he is someone who mates less often or more often than average. If such males are in a situation where they can care for offspring, then those whose future likely mating success is higher should probably care less.

36. B: You've assumed that there is a trade-off between care and mate attraction. Which isn't so clear for us gobies, you know.

37. A: Yes, but I'm making a general point. It could also be that males know nothing about their relative mating ability except for the fact that they have mated. (They can't make caring decisions if they do not mate.) But selection will, over evolutionary time, realize that mated males are not random males.

38: B: Surely that's not true if mating is random.

39: A: Yes, and what I'm going to say now relies on non-random mating. Such a thing is likely to happen for the mate-limited sex. If, for example, only large males mate, then there is non-random variance in mating success.

40: B: Why is variance important if the mean reproductive success is governed by the equality of the two sexes? Let me think... ah, now I see: because if there is variance, then there are probably many males with zero 


\begin{abstract}
or little success, and the successful ones, who find themselves with females, have better future prospects than the average male.

41. A: Yes! So males favoured by sexual selection can actually reproduce more often than females, as they are a particularly successful subset of the population; so any male who has mated should be quite optimistic about his future success with other females. Even if they can't perceive their success relative to other males, selection makes sure that earlier deserters are rewarded as their mating success is, like I said, elevated in a non-random way.

42. B: I'd still say it's different for us gobies - females actually like those males who care.

43. A: Sure, there's diversity in nature. Fish in general are a bit like that. 44. B: I seem to remember I had another point initially... Ah yes, point 10: this whole argument seemed to rest on the assumption that there are equally many males as females. But there's often mortality and lifespan differences between the sexes. Sometimes there are even biases in the primary sex ratio, or at least in the sex ratio at maturation.

45. A: Actually, the researchers I've been listening to are quite excited about this. If there's very many males per female in the population as a whole, then many more males must find it harder to find a mate than when the sex ratio is unbiased.

46: B: So for mated males to be optimistic about their future success with other females (who are now few and heavily competed over), there should be really strong sexual selection so that only a very small subset of males mate. If sexual selection is any less strong, then males will be pessimistic about future mating success.

$47 \mathrm{~A}$ : Indeed! Now you are starting to talk like a scientist. So, if the adult sex ratio is male-biased, males should probably stay with their current young for longer than when the adult sex ratio is female-biased.

48: B: I can see what you're getting at. The male-biased case is a 'bird' scenario where males, being numerous, have trouble finding a female to pair with - so deserters can't be that successful, and it's good to spend effort on one's offspring (even if one is happy to mate with other females should opportunities arise). And the female-biased adult sex ratio is like that in many mammals, where it would be rather daft for a male to forego all new breeding opportunities by staying monogamous. Or at least the benefits should be really substantial for this to happen.

49: A: Yup. Like if biparental care is much more efficient at keeping offspring alive than uniparental one. All things interact, of course. 50: B: Indeed. But I keep wondering where these sex ratio biases come from. Didn't you say that Fisherian sex ratio selection typically predicts unbiased primary ratios.

51: A: True, but it does nothing to prevent sex ratios from getting biased later in life. If, for example, caring for offspring is much more dangerous than competing for mates, and females do more caring, then we get a male-biased populations.

52: B: That creates the 'bird' scenario.

53: A: Yes, and if competing is the dangerous activity, and males do it more, then we get female-biased populations. If only relatively few males survive then for the remaining ones it must be really lucrative to return to the mating pool to find females as soon as they are back in the receptive state.

54: B: That's rather nice given that mammal populations tend to have a female-biased adult sex ratio and no male care. But I still keep getting confused. I would engage less in an activity if it got more dangerous. 55: A: It is counterintuitive, but in an intriguing way. It shows that the potential rewards - the number of females per survivor - can increase faster than the costs - the potential that you die in the competition. It really can happen when both females and males adjust their behaviour to the risks.

56: B: I would never have predicted that, until you told me about the feedbacks with population-wide sex ratios.

57: A: Which probably shows that it's important to consider these feedbacks between individual success and population-level phenomena, at least for long-term evolutionary scenarios.

58: B: But surely there's phylogenetic inertia too. Male mammals don't suddenly start behaving like male birds. They are unable to lactate, so it's less clear how they could do something useful for the offspring.

59: A: Point taken. There is probably phylogenetic inertia and taxonspecific details everywhere. That's biology. But I still think it's good to look for generalities. Birds with mammal-like mating systems also tend to have mammal-like adult sex ratios.

60: B: I agree, that's a nice finding. But I must return to the issue that the whole logic of what you've told me rests on the notion that care and competition are flipside activities - you can't do both simultaneously. That's just not true for us gobies.

61: A: I know, I'm a goby too. The researchers say there are many things that could be tweaked to produce various special cases like ourselves. Indeed, they seem to want further work done to examine which other factors really do vary across species or taxa, because they have this model that they'd like expanded. That way we can get a better picture of what explains the special as well as the common cases.

62: B: Why didn't they do it here?

63: A: I think they decided it was time to stop talking and start writing.
\end{abstract}

interdependence of male and female rates of reproduction has been integral to studies of primary sex ratio evolution for over a century (Düsing, 1884; for a translation see Edwards, 2000; Fisher, 1930). It is therefore surprising that the consequences of the Fisher condition for the causal path from anisogamy to sex roles have been largely neglected.

We will argue that to understand fully sex role evolution the influence of the ASR must be considered. This is obvious given the caveat that the sexes' reproductive rates are only identical when the ASR is even. Trivers (1972) rightly noted that as parental investment differences evolve this can lead to sex-specific mortalities, which alter the numbers of individuals of each sex competing for mates. Empirical data show that these changes in ASRs can be substantial. Trivers (1972) encouraged others to explore the implications of biased ASRs but his call went largely unheeded, and subsequent studies have focused on OSR (ratio of sexually active males to receptive females) instead. We will therefore explore in detail how the ASR influences sex role evolution.

Finally, an important simplification in several subsequent mathematical models that built on Trivers' main thesis, has been that parental investment patterns affect the OSR, but there is no feedback in the opposite direction. There are a few theoretical studies that address this feedback (e.g. Grafen \& Sibly, 1978; Houston \& McNamara, 2002, 2005), but intriguingly they do not place their results in an OSR framework. Our model incorporates the relevant feedback and explicitly relates sex roles to evolution of both the OSR and ASR. 
In sum, our integrative mathematical model shows that including feedback from population-level characteristics is essential to make sensible evolutionary predictions about sex roles. Our model explores interactions among several phenomena that can alter parental investment: offspring need, multiple mating, sexual selection (i.e. non-random variance in mating success) and sex-specific mortalities with their consequences for population sex ratios. Some of our results are surprisingly counterintuitive. For example, the 'eagerness' of individuals of a given sex to perform an activity (competing or caring) can increase if the mortality associated with this task increases. This finding seems paradoxical but the available comparative data from birds and mammals support it. Finally, we conclude our review with some caveats about our model. We highlight the need for specific empirical tests and data sets and suggest future directions for theoretical research.

\section{Trivers' original verbal model and its hidden problems}

For some, the evolution of sex roles was satisfactorily explained several decades ago. Trivers (1972) presented a classic account of parental investment and sexual selection that has formed the basis for textbook accounts and classroom lectures on sex role evolution ever since. We will argue, however, that some essential factors were missing from this account. This has led to verbal arguments that reach the right conclusions for the wrong reasons.

Trivers' (1972) argument hinged on anisogamy, i.e. that females by definition produce larger gametes than males. His most important conceptual insight, which built on work on life history trade-offs by Williams (1966), was that there is often a difference between the sexes in parental investment (use of resources which increase current offspring survival at a cost to investment in future offspring by the parent). Trivers pointed out that parental investment is, by definition, costly to a parent and outlined various ways in which the costs and benefits of caring and competing then become sexspecific. (In this review we use 'care' as a shorthand for post-mating parental investment, so we exclude behaviours that incur no fitness costs.)

Trivers noted that sex-role reversal, where females compete for mates more strongly than males, is associated with greater male than female parental investment (but see Sogabe \& Yanagisawa, 2007). He then linked parental investment to conventional sex roles by pointing out that gametes are a form of pre-mating parental investment. Females, because they produce eggs, make a larger parental investment prior to mating. Males can replenish their gamete supply and return to the mating pool sooner than females because they produce small, cheap sperm rather than large, costly eggs. Strictly speaking, these statements about rates of recovery after mating should be framed in terms of the total time and energy costs associated with a mating event, rather than the production of individual gametes (Baylis, 1981). Even so, male ejaculates tend to represent a smaller total gametic investment per mating than the eggs that a female releases.

When there is no post-mating parental care, and an even ASR, anisogamy clearly predicts that an excess of males will compete for a limited supply of females. In modern jargon, the higher potential reproductive rate (PRR) of males creates a male-biased OSR (Clutton-Brock \& Vincent, 1991; Clutton-Brock \& Parker, 1992). This increases the variance in male mating success and the opportunity for sexual selection. Males are then forced to compete more intensely for access to mates, generating sexual selection for traits that increase male mating success. It is incontrovertible that Trivers correctly identified a causal link between greater pre-mating parental investment by females and stronger sexual selection on males. But does that link tell the whole story?

\subsection{How do you go from anisogamy to female-biased care?}

The real problem is accounting for the generally positive relationship between pre-mating and post-mating parental investment. Why does anisogamy lead to the evolution of female-biased parental care? Trivers (1972) is still widely invoked to explain this phenomenon, which we call sex role divergence (a shorthand for a tendency for initial investment asymmetries to amplify in a self-reinforcing manner, until distinct sex roles are established). Trivers put forward two arguments. One essentially strengthened the case that females should invest heavily into parental care, the other emphasizes factors that diminish the likelihood that males invest. Colloquially, these 'female' and 'male' arguments could be called the 'can't afford to lose past investment' effect and the 'I compete, I don't have time to care' effect.

\subsection{The female argument: 'can't afford to lose past investment'}

Trivers (1972) states: 'Since the female already invests more than the male, breeding failure for lack of an additional investment selects more strongly against her than against the male'. Unfortunately, this argument appears to commit the Concorde fallacy - the cognitive illusion that past investment per se makes it more profitable to continue with, rather than abandon, a costly activity (Dawkins \& Carlisle, 1976). That past investment alone is irrelevant to decisions about the future is illustrated by the observation that a female should, in principle, be indifferent about whether she cares for a newborn baby brother or her own newborn child. Despite a greater initial investment in her own child, she is equally closely related to both individuals and the future pay-offs from caring are identical 
(Dawkins \& Carlisle, 1976). The weakness of the past investment argument is even starker when one notes that a parent's total investment in a brood can only increase with time. A decision based purely on past investment would lead to the conclusion that a parent should never abandon the current brood.

The female argument can, however, be resuscitated if it is phrased in terms of future benefits rather than past investment. This circumvents the logical fallacy, but we do not think it resolves the biological problem. There are at least two such modifications to the argument and neither is convincing. The first focuses on how PRRs alter future reproductive options. The second considers the effect of past activity on an individual's current state. In both cases, it is important to focus on factors that differ between the sexes with respect to their future activities if they either stay with or desert their young.

\subsubsection{The first resurrection attempt: what happens when pre-mating investment changes potential reproductive rates?}

Every time an offspring dies due to a reduction in parental care it is detrimental to the fitness of both parents. But given different future options, is the loss equally important to both? It is tempting to argue that because of anisogamy females will take longer to compensate for this loss than males, who can produce offspring elsewhere at a higher rate. Reproductive opportunities are constrained by a parent's own PRR (Clutton-Brock \& Parker, 1992). A naïve interpretation of this is that the lower PRR of females means that their future reproductive rate is lower than that of males, making females less inclined to desert. Unfortunately, this argument ignores the basic fact that reproductive opportunities depend on the availability of sexual partners (Parker \& Simmons, 1996). Queller (1997) presented the first general argument for sex differences in parental investment that took this constraint into account. He noted that sex ratios affect the future success of a deserting parent. Whichever sex is currently rarer in the population has a higher per capita reproductive success. No other relationship is possible in a population of diploid, sexually reproducing individuals because every offspring has one father and one mother. This so-called 'Fisher condition' has important consequences for sex roles (Houston \& McNamara, 2005; Kokko et al., 2006). If females have a low PRR, the OSR will be male-biased. Males could reproduce faster in principle, but assuming that all males fulfil their potential violates the Fisher condition.

How detrimental is the difference between potential and actual reproductive rates to the female argument? To find out, we must examine how long a parent should stay with its offspring. As parental investment accumulates and offspring approach independence, the marginal benefit of caring diminishes. The benefit can be expressed as an increase in offspring reproductive value (Fig. 1), typically summarized as an increased probability that an offspring can survive on its own and enter the breeding population. Trivers, himself, produced several graphs that
Benefits of care

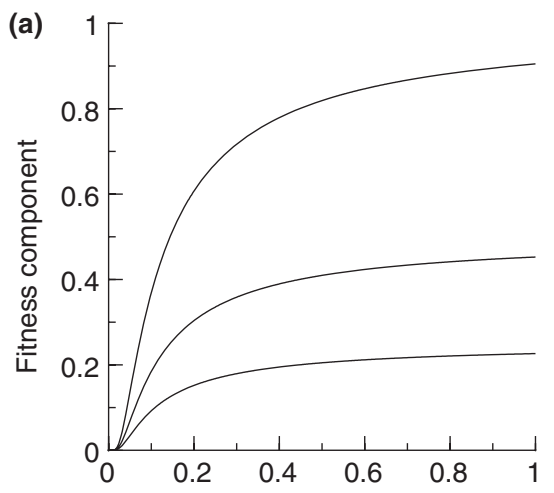

Marginal benefits

(b)

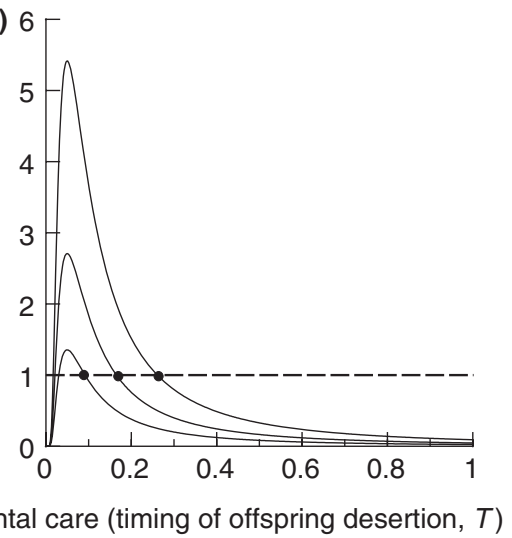

(c)

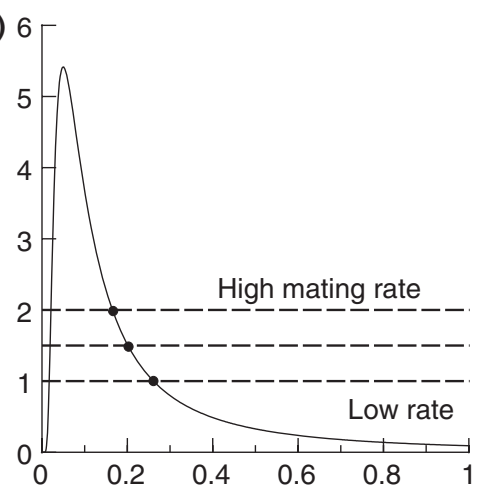

Fig. 1 (a) The likely fitness gains for a parent equal the sum of reproductive values of all offspring in a set $\times$ average relatedness to them (which depends on the degree of multiple parentage). These fitness gains saturate with time spent caring as offspring become less dependent on their parents. Curves obey, from top to bottom, $S\left(T_{\text {total }}\right)=\exp \left(-\alpha / T_{\text {total }}\right), S\left(T_{\text {total }}\right)=\exp \left(-\alpha / T_{\text {total }}\right) / 2$, and $S\left(T_{\text {total }}\right)=\exp \left(-\alpha / T_{\text {total }}\right) / 4$, all with $\alpha=0.1$. The different curves can either be read to imply that offspring survival prospects vary, or they can be interpreted as smaller benefits resulting from lower parentage: in the latter case, offspring survival remains at $S\left(T_{\text {total }}\right)=\exp \left(-\alpha / T_{\text {total }}\right)$ but one, two or four individuals of the same sex as the parent have contributed to the brood. (b) The corresponding marginal benefits. Parental care evolution can only reach a stable equilibrium in the decreasing part of the curve. The horizontal dashed line indicates a hypothetical level of expected offspring production when a parent returns to the mating pool. Parental investment evolves to lower levels (dots) with diminishing expected genetic relatedness to the brood or weaker effects of care on maximum offspring survival. (c) For parents with better mating prospects after desertion (higher horizontal lines), the predicted care levels are lower (dots). 
made this point with great clarity. By definition there is a trade-off between current parental investment and future reproduction by the parent. A parent should stop investing in its current offspring when the marginal gain from caring is less than that from pursuing other activities that enhance the parent's future reproductive opportunities.

But which parent should stop first? If all else is equal (e.g. both parents are equally capable of providing care) and the only asymmetry between the sexes is that males are mate-limited while females are not, then it is males who are selected to provide more care (Fig. 1). Clearly, the first version of the female argument fails dismally. It predicts egalitarian care, not divergent sex roles. We will repeatedly return to this prediction.

\subsubsection{The second resurrection attempt: what if past} investment influences current costs and benefits of caring? What if all else is not equal between males and females? Parental care ends when the marginal gains from further care equal those from attempting to breed again (which might require a 'time out' period during which energetic and gametic resources are replenished) (Fig. 1). Leaving aside sex differences in mating rate, why would the cost or benefit curves differ between the sexes? Specifically, how could the greater initial investment of females into gametes lead to a sex difference in the future pay-offs from caring so that females have a smaller incentive than males to desert?

We can invoke past investment to explain decisions about the future without committing the Concorde Fallacy, if past investment alters current pay-offs. For example, a fish might invest more into guarding fry that have already received care than a clutch of recently laid eggs if this past parental investment elevates the probability that a fixed amount of additional care will yield independent offspring. This simply requires that over some range of investment, the rate of return per additional unit of care is an increasing function of the total prior investment (Coleman et al., 1985; Lavery $\&$ Keenleyside, 1990; Ackerman \& Eadie, 2003).

There is abundant empirical evidence from life history studies that past investment reduces a parent's residual reproductive value (Roff, 2002). This can generate a correlation between past investment and future breeding decisions so it has been suggested that the term 'Concorde fallacy' is misleading in the context of parental care (Coleman \& Gross, 1991; Gross, 2005). However, being explicit about future benefits does not resolve the problem that we still need to identify a general mechanism whereby females, because they make a greater parental investment prior to mating, have increased future pay-offs for the same amount of parental care in the current offspring relative to those of males (Sargent $\delta$ Gross, 1985; Dawkins, 1989 [p. 150]). (For sex-specific differences in the pay-offs from desertion see Section 2.3.)
Producing eggs rather than sperm could decrease the relative costs and/or increase the benefits of parental care. Either mechanism could generate a positive link between past investment and current pay-offs:

(a) Past investment reduces the current costs of care. It is difficult to visualize a general principle that would generate this relationship. In fact, it is more readily conceivable that the greater cost of egg production places females in a physiological state where the absolute and possibly the marginal costs of subsequent caring are greater than those for males. For example, the costs of caring could be an increasing function of a decline in body condition or energy reserves. There is also evidence in many taxa that investment in somatic growth yields greater returns for females than for males, e.g. when fecundity increases with size (Blanckenhorn, 2005). In such cases, females will actually pay more than males when they invest resources in care rather than in growth (Sargent \& Gross, 1993; Balshine-Earn, 1995). This reduces the likelihood of female care. Indeed, this very argument is used to explain why males provide more than females in many egg-laying fish (Sargent \& Gross, 1993).

(b) Past investment increases the current benefits of care. The benefit of care is to improve offspring fitness. This benefit might differ between the sexes if a parent's current body condition or energy reserves affect the quality of care it provides. As noted above, however, it is difficult to see why the greater past investment of a female, which a priori should lead to lower female than male body condition, selects for greater female care (except in exceptional or restrictive circumstances such as terminal investment).

There are clearly unresolved problems with the 'can't afford to lose' argument. In the most naïve form it is based on a fallacy. If one constructs a more sophisticated argument invoking life history principles that connect past investment to the current reproductive value of a parent, it is still unclear why this would consistently favour female care. For example, studies of sexual dimorphism show that investment in somatic growth can benefit females more than males or the exact reverse (Fairbairn et al., 2007). It is inappropriate simply to assume that the sexes systematically differ in the absolute costs of caring because it yields the desired outcome (for an analogous argument about mate searching see Kokko \& Wong, 2007).

\subsubsection{Uncertain parentage provides another argument} for why a set of offspring are more valuable to a female There is one way in which the current benefits of caring systematically differ between males and females. This is due to uncertain parentage because of sperm competition, which selects for reduced male care (Trivers, 1972, 1985; Yamamura \& Tsuji, 1993; Queller, 1997). Unfortunately, the validity of this claim is occasionally disputed because within and across species predictions are conflated. 
There is a distinction between short-term responses to loss of paternity and long-term evolution of male care. A male should only show a behavioural response to lower paternity by reducing care if he can expect higher paternity in future broods (Westneat \& Sherman, 1993; Wright, 1998; Sheldon, 2002). This depends on whether paternity changes with age, size or status or is purely stochastic, as well as other unusual biological features (e.g. García-González et al., 2003). This might explain why within species, paternity is positively correlated with levels of male care in some species (e.g. Hunt \& Simmons, 2002; Neff, 2003), while the relationship is weak or absent in others (e.g. Rios-Cardenas \& Webster, 2005).

In contrast, over evolutionary time a decline in mean paternity should reduce the mean level of male care. There is some empirical support for this claim (Møller $\delta$ Cuervo, 2000). This pattern is predicted because, whenever there is mixed paternity, males must have reproductive opportunities outside those with their social mate. This increases the incentive to seek out additional mating opportunities, while the benefit of ongoing care simultaneously decreases with declining parentage within each brood (Yamamura \& Tsuji, 1993; Queller, 1997). Whenever there is a trade-off between current care and obtaining extra-pair matings (Dawson, 1996; Magrath \& Komdeur, 2003), the net effect is selection for males to desert sooner or otherwise provide less care. This generates a positive relationship between paternity and care across species.

That females lose less parentage than males is only tenuously connected to the 'can't afford to lose' argument. Of course females have more to lose if they are the genetic parent of more offspring per brood. Seen from the male perspective, however, the statement that he can afford to lose current offspring that are not his own is very distant from the original arguments that directly relied on a causal effect of the greater past investment of females in gametes.

\subsection{The male argument: 'I compete, I don't have the time to care'}

Trivers' second insight was that 'male-male competition will tend to operate against male parented investment, in that any male investment in one female's young should decrease the male's chances of inseminating other females' (Trivers, 1972). This argument is not confined to time costs. It implicitly assumes that the best response for males, who face more mating competitors than females, is to invest more heavily in weaponry, ornaments or other traits that increase their access to mates. There is, however, a valid counterargument: when the going gets tough, the smart do something else. More formally, the question becomes: are the marginal gains from increased investment in competition really greater than those from caring more for offspring?
Intriguingly, intense male-male competition is sometimes invoked to explain why males avoid competing for mates and become monogamous (Fromhage et al., 2005). For example, Segoli et al. (2006) suggested that male white widowspiders (Lactrodectus pallidus) invest heavily into mating with a single female (often losing the tips of their copulatory organs) because intense male-male competition reduces the likelihood that they will obtain another mate (see also Fromhage et al., 2005). It is perfectly reasonable to ask why a male would return to compete for mates when it is difficult to succeed, if he can instead profitably care for and/or ensure greater paternity in the current brood. Although males often do invest more in competitive traits in species when competition is intense (e.g. Weckerly, 1998; Byrne et al., 2002; Lindenfors et al., 2002; Bro-Jørgensen, 2007), something is amiss if biologists can use the same logic to reach two diametrically opposed conclusions. What is obviously missing in many models of sex role evolution is an explicit description of the alternate routes to increased fitness that a male (or female) can pursue when the OSR is biased in their direction, other than investing more in being competitive.

\subsubsection{An assumption is not an explanation}

If the only option for a male faced with a male-biased OSR is to increase his investment in competitive traits, then sex role divergence is explained. However, this is an argument that artificially constrains the outcome and simply confirms the original prejudice. There is a recurring hidden assumption in many models that the sex that provides less care must automatically evolve to provide even less. In short, sex role divergence is assumed rather than explained (see Dawkins, 1989, p. 301 for an example of the seductive power of this line of argument). Given that male care has repeatedly evolved, it is more reasonable to ask what else a male in a competitive situation can do to increase his fitness. The most obvious alternative for males with offspring is to provide more parental care. Therefore a useful model of sex role evolution must ensure that each sex can decide whether to continue to care or seek a new mate.

\subsubsection{Frequency-dependence: the Fisher condition is analogous to Fisherian sex ratio evolution}

If the OSR is male-biased, mate-searching males will take longer than mate-searching females to find a mate. This reiterates the counterintuitive conclusion that we reached for the first version of the female argument. A male should invest in his current offspring for longer than his mate because, upon desertion, he faces a longer wait than her before reproducing (Fig. 1). More generally, selection favours any activity that is more profitable than immediate engagement in an increasingly competitive situation.

Frequency-dependent selection on care is analogous to that which explains offspring sex ratios (Fisher, 1930). If 
one sex is overrepresented in the primary sex ratio, it is profitable for parents to shift reproductive effort from producing offspring of this sex (because these will have low reproductive success) to another activity with larger fitness returns, in this case to producing the underrepresented sex. In the context of care, one sex can be overrepresented in the mating pool because it provides less care. It is now adaptive for members of this sex to react to their own low prospects of reproductive success and shift effort towards another activity when possible. A clear alternative arises when they have mated and are in the position to provide care. Thus members of the less caring sex should delay their return to the mating pool and evolve to care for longer. The analogy is clear (the decision of parents to produce sons or daughters is driven by selection analogous to that on the decision of mated individuals to care or desert). Just as Fisherian sex ratio selection pushes primary sex ratios towards unity, the Fisher condition shifts OSRs towards equality by selecting for more egalitarian care (we will confirm this surprising result mathematically). Sex role divergence should not be taken for granted. Given frequency-dependent selection it is an evolutionary conundrum.

\section{How past assumptions have shaped subsequent research}

Numerous mathematical models have built on Trivers (1972) to investigate specific, isolated aspects of sex roles. A non-exhaustive list of questions that have been addressed include whether a parent should desert its offspring (Maynard Smith, 1977; Yamamura \& Tsuji, 1993; Webb et al., 1999; Wade \& Shuster, 2002; Fromhage et al., 2007); how trade-offs between parental effort and mating effort influence care decisions (Dawson, 1996); how much care each sex should provide if there is biparental care (Houston \& McNamara, 2002); which sex should invest more in being choosy (Owens \& Thompson, 1994; Johnstone et al., 1996; Kokko \& Johnstone, 2002); how male care coevolves with female mate choice (Mylius, 1999; Wakano \& Ihara, 2005; Seki et al., 2007); and which sex will compete more strongly for mates (Clutton-Brock \& Parker, 1992; Parker \& Simmons, 1996; Kokko \& Monaghan, 2001). Taken together, these models contain all the elements needed to build a general model. Individually, however, they either deal with taxon-specific scenarios, fail to report variables of interest for other models or make assumptions that prevent some key processes from operating. Consequently, it is too onerous a task to integrate the existing models' insights to obtain a complete picture of sex role evolution. We will nevertheless use them to highlight key findings, before presenting our own integrative model.

The problematic aspects of Trivers' (1972) presentation have been repeated to varying degrees in most subsequent mathematical models. In general, however, the 'female argument' is not invoked because formal models explicitly calculate fitness. We are, in fact, unaware of any mathematical models that simply assume that past investment correlates with current activities. In contrast, many verbal accounts of sex role evolution do little more than cite Trivers' statement that females make a greater parental investment than males in gametes and then, as an aside, note that the same is often true for investment in parental care. They provide no explanation for the link between past and present investment. This implies either that the Concorde Fallacy continues to trap the unwary, or that the causal link between egg production and greater female care is still unclear to many authors.

Fortunately, recent textbooks show a welcome trend towards greater clarity. For example, in the 1998 edition of his excellent textbook, Alcock (1998) describes anisogamy and its effect on rates of reproduction and then simply notes: 'These differences between the sexes in gametic parental investment are often amplified in other kinds of parental investment, which include the food or protection parents sometimes give their progeny'. The 2005 edition, in contrast, makes readers aware of the pitfalls inherent in the female argument in a section entitled 'Why more care from mothers than fathers?'

\subsection{Using the OSR: avoid oversimplification, allow feedback with care and count properly}

A weakness of many mathematical models of sex role evolution is the assumption, implicit in the verbal 'male argument', that the OSR predicts which sex will compete more strongly (Emlen \& Oring, 1977; Clutton-Brock \& Parker, 1992; Kvarnemo \& Merilaita, 2006). The link between the OSR and the intensity of mate competition is an assumption, not a logical necessity. All models require simplifying assumptions, but this can be considered an unsatisfactory one. Empirical studies show that, in some cases, the relationship is nonlinear, absent or even negative, especially when confounding parameters such as population density covary (for a review see Kokko \& Rankin, 2006). For example, in the European bitterling fish (Rhodeus sericeus) large males are territorial and most spawnings involve a single breeding pair. When the male population density is high, however, large males abandon territoriality and compete with smaller males in group spawning. This reduces the opportunity for sexual selection on male size so that a more malebiased OSR is associated with a decline in the intensity of sexual selection (Mills \& Reynolds, 2003). In common lizards (Lacerta vivipara) experimental manipulations show that a switch from a female to male-biased ASR (a manipulation that forces the OSR to change in the same direction) led to a marked decrease in sexual selection on male body size (Fitze \& Le Galliard, 2008).

More generally, the OSR is an imperfect predictor of the Bateman gradient (the relationship between the 
number of matings and fitness, Bateman, 1948). It is consequently better to explicitly quantify how mating rate affects fitness, which is by definition the Bateman gradient (Bateman, 1948; Arnold \& Duvall, 1994; Lorch, 2002). Models that adopt this approach show that the benefits of an increased mating rate are sometimes larger (i.e. the Bateman gradient is steeper) for the rare, matelimiting sex in the OSR. For example, this can happen when the sexes have different life histories because mortality rates differ between caring and competing for mates (Kokko \& Monaghan, 2001). More generally, as our integrative model will show, the OSR assumption is deeply unsatisfying because the ASR has an independent effect on the value of competing for mates.

Even models that avoid equating a male-biased OSR with conventional sex roles (e.g. Kokko \& Monaghan, 2001) can still fall prey to another implicit and widespread modelling assumption: parental investment influences the OSR, but changes in the OSR do not affect how much each sex invests in care (e.g. Clutton-Brock $\delta$ Parker, 1992; Owens \& Thompson, 1994; Lorch, 2002). This precludes reciprocal feedback between the OSR and sex-specific parental investment. This simplification has largely gone unnoticed by empiricists, because their primary goal is usually to explain which sex competes or cares when sexual differences in parental investment are already well established. For example, models that exclude feedback perform well when short-term fluctuations in the environment affect how long it takes each sex to accrue sufficient resources to reproduce or to rear a brood to independence. The consequent effects on PRRs and the OSR occur almost immediately when compared with any longer term changes in investment decisions. This means that certain terms in the equations of theoretical models remain largely unchanged while others vary. Whether this is a strength or weakness depends on the time scale of the investigation.

To make long-term evolutionary predictions about care patterns, feedback with the OSR must be taken into account. In the last decade a series of perhaps underappreciated papers have raised awareness that the Fisher condition matters: the relative benefits of deserting cannot be calculated unless the number of potential mates and competitors are correctly identified (Queller, 1997; Webb et al., 1999; Wade \& Shuster, 2002; Houston et al., 2005; Fromhage et al., 2007; Seki et al., 2007). Failure to do so is a surprisingly easily committed clerical error (see Fromhage et al., 2007 on Wade \& Shuster, 2002). For example, Maynard Smith (1977) in an otherwise insightful model simply assigned fixed values for the benefits of deserting a mate (see Houston $\delta$ McNamara, 2005 for a discussion). As Trivers (1972) pointed out, a useful model of sex role evolution is largely a question of working out when individuals should desert their offspring. Decisions about parental investment directly affect the OSR even if various activities (competing for mates, or investing in offspring) do not differentially affect adult mortalities. If they do the situation becomes even more complex because sex role evolution will then influence the ASR and the OSR will additionally respond to this population-level change in ways that we will explain with our integrative model.

\subsection{The OSR and empirical studies: phenotypic plasticity is not a test of how care evolves}

Several empirical examples illustrate the importance of testing models over an appropriate time scale. For example, in an Australian bushcricket males take longer to produce a spermatophore in a low food environment, so the OSR becomes female-biased and females compete for males (e.g. Gwynne, 1981, 1990; Gwynne \& Simmons, 1990). In many studies, the OSR is changed through an experimental manipulation (e.g. Keenleyside, 1985a, b; Balshine-Earn \& Earn, 1998) or by sudden sex-biased mortality (e.g. Richardson, 1987). The rarer sex in the newly created OSR often responds by reducing care levels and deserting current mates (although it is worthy of note that the OSR bias is usually shifted towards the sex with the greater propensity to desert based on natural observation, rather than equally often in both directions). This change in care appears to violate the assumptions of theoretical models that do not permit feedback. In practice, however, the period over which breeding is monitored is sufficiently short that any change in the time each sex spends caring does not substantially alter the OSR (which is defined by the starting conditions).

The crux of the issue is that documenting phenotypic plasticity in care behaviour is not the same as being able to predict the course of evolution. To take a specific example, Keenleyside (1983) experimentally increased the availability of females in closed populations of a cichlid with biparental care. This resulted in males deserting their broods sooner. There was, however, no investigation into the long-term effect of male desertion on the OSR. Now consider an evolutionary scenario where female availability remained constantly high, e.g. because some novel environmental factor reduced female mortality. In this case, as in the experimental study, males would be expected to desert prematurely. However, males would eventually face tougher competition for mates as their tendency to desert becomes established in the population. This competition might even be amplified if females that care alone incurred greater energetic costs, which delayed their return to the mating pool. If female-only care increased the female mortality rate it might even mitigate the original decline in mortality. In short, adding females or removing males from an experimental population can only test for plasticity in care behaviour. These changes might bear no resemblance to long-term evolutionary shifts in patterns of parental investment or mating competition. It is also worthy of note that these kinds of experimental 
studies simultaneously adjust the ASR and OSR. This conflates simple interpretation if the two ratios have independent effects over evolutionary time scales.

The rules governing evolutionary patterns over broad taxonomic scales encompass a larger set of interacting parameters than those affected by plastic responses to short-term variation in the environment. In the short term some parameters can be treated as fixed because interactions between parameters are low. This is why plastic changes in parental decisions or responses to mating competitors can often be related to a single parameter (usually the OSR) with relative ease. Simmons \& Kvarnemo (2006) provide an illuminating example and then discuss why changes in the OSR are more likely to affect sex roles in the short term than the costs of breeding, even though the latter are predicted to have a major effect on the long-term evolution of the direction of sexual selection (Kokko \& Monaghan, 2001).

\section{Progress towards solutions}

So far, we have simply concluded that sex role divergence is a conundrum once the equalizing effect of the Fisher condition is taken into account. So how can sex roles diverge? In this section we describe the factors that we consider key to building an integrative model of sex role evolution. We point out that verbal arguments become increasingly difficult to sustain when multiple factors interact. In Section 5 we therefore explain the approach we have used to incorporate these factors into a formal mathematical model.

\subsection{Multiple mating and sperm competition influence parentage}

Earlier (Section 2.2.3.) we mentioned that reduced parentage in a given set of offspring due to multiple mating by females selects against male care. This explanation is powerful and widely applicable (Yamamura \& Tsuji, 1993; Queller, 1997). Sperm competition is a near-automatic consequence of anisogamy if the two sexes are equally common and have similar total energy budgets on gamete production, and population densities are such that sperm from several males compete for each egg. It can be ignored when females are strictly monogamous, but this is rare in nature (Jennions \& Petrie, 2000; Griffith et al., 2002). It also does not apply in some marine species where sperm limitation is a problem (Levitan \& Petersen, 1995; Levitan, 2002), but in these species there is also no parental care.

\subsection{Sexual selection and mating prospects: whose care decisions are exposed to selection?}

Sexual selection, which we here define as non-random variance in mating success, is another widespread process that is likely to contribute to determining parental investment and sex roles. Queller (1997) pointed out that some males have greater access to mates than others in the face of sexual competition. Given sexual selection, some males must possess traits that elevate their mating success above the level implied by the Fisher condition. These sexually selected traits, that can act pre- and/or post-copulation, improve male reproductive success in situations involving female choice and/or male-male competition. In Fig. 1 this corresponds to some males enjoying higher expected reproductive opportunities after deserting their offspring.

If male traits must exceed a threshold value to ensure mating success, only some males are 'qualified to mate' (sensu Ahnesjö et al., 2001). More importantly, however, only males that mate can sire offspring and selection can only act upon the parenting traits of these reproductively successful males. This tends to lower male parental investment in the population at large because genes for reduced care become visible to selection in males that have mating rates higher than the population average. These genes therefore contribute disproportionately to subsequent generations. All this process requires is non-random variance in male mating success that is greater than the corresponding female variance. Males (or females) do not need to show phenotypic plasticity and adjust their care behaviour based on a perception of their future mating prospects.

There is empirical evidence that males with higher expected future reproductive success provide less parental care, albeit due to behavioural plasticity (e.g. Robertson \& Roitberg, 1998; Jennions \& Polakow, 2001; Duckworth et al., 2003; Mitchell et al., 2007). It should be noted, however, that there are also species in which more attractive males, who presumably have higher mating rates, provide more parental care (for a review see Møller \& Jennions, 2001).

The fact that stronger sexual selection on males than females selects against male care resurrects the credibility of the 'male argument' to some extent. Nevertheless, it remains incorrect to simply assume that a male-biased OSR creates sexual selection which, in turn, selects against male care. There are important caveats. First, this conclusion is obviously reversed when male care is the sexually selected trait, as is the case when female choice is based on male parenting ability (Hoelzer, 1989). Second, and more importantly, the net direction of selection is affected, but cannot be solely predicted, by the OSR. Consider, for example, a population with an OSR of 10:1 (males : females). Frequency-dependent selection for male care will be opposed by sexual selection on males, but this has to be very strong to overcome the Fisher condition so that net selection favours earlier male desertion. Specifically, the future expected mating rate of a currently mated male must be at least ten-fold greater than that of the average male that contributes to the OSR. 


\subsection{Which sex ratio?}

An intriguing omission from Queller's (1997) excellent account of sex role divergence is that he did not state whether he was referring to the ASR or the OSR. This is noteworthy because the OSR has been assigned a far more prominent role in sex role theory (e.g. CluttonBrock \& Parker, 1992; Simmons \& Kvarnemo, 2006). For example, a 'topic' search in Web of Science shows that the ratio of papers with the term 'operational sex ratio*' to 'adult sex ratio*' that include the term 'parental care' is $3: 1$ and for 'sexual selection' this rises to $4.5: 1$. The ASR is often treated as a nuisance variable that is only needed to calculate the OSR correctly (e.g. Clutton-Brock \& Parker, 1992; Kokko \& Monaghan, 2001; Kvarnemo \& Merilaita, 2006). Empiricists have likewise expended far more energy calculating the OSR than the ASR (Donald, 2007; but see Le Galliard et al., 2005).

There is a general tendency to equate the ASR and OSR. One possible reason is the fact that in most experimental studies both ratios are manipulated simultaneously because the starting condition is one where all adults contribute to the OSR. For example, $X$ females and $Y$ males are placed in an enclosure and the reproductive variable of interest is then measured. Variation among enclosures is then attributed to variation in $X: Y$ which is, however, almost always labelled as the OSR rather than ASR (e.g. Alonso-Pimentel \& Papaj, 1999; Jirotkul, 1999; Klemme et al., 2007; for an exception see Fitze $\delta$ Le Galliard, 2008).

\subsubsection{Can the ASR have an independent influence on sex roles?}

Given an even primary sex ratio, the ASR is determined by differences in mortality arising from sex role divergence that affects investment in traits favoured during mate competition or caring (e.g. Promislow, 1992; Promislow et al., 1992; Liker \& Székely, 2005; Benito \& González-Solís, 2007) and in other life history traits (e.g. Moore \& Wilson, 2002; Tschirren et al., 2003). Despite this, feedback between the ASR and patterns of parental investment has been neglected. Some researchers have suggested that the ASR is a major factor in sex role evolution but their ideas have not been incorporated into mainstream theory. For example, Breitwisch (1989) speculated that a male-biased ASR in birds allows females to 'extract' more male parental care, and at least three theoretical studies report that male care evolves more easily in male-biased populations (Yamamura $\&$ Tsuji, 1993; McNamara et al., 2000; Houston \& McNamara, 2002). Donald (2007) noted that bird species with little or no male care seem to have female-biased ASRs, both being typical mammalian features. In contrast, in birds with biparental care, the ASR seems to be male-biased (Donald, 2007). In fish, Sogabe \& Yanagisawa (2007) and Forsgren et al. (2004) have attributed sex-role reversal to the ASR becoming female-biased. Spiders, despite lacking paternal care, provide intriguing data on a related trait. Cases where male mating investment is confined to a single female (monogyny) are more common when sex ratios are male-biased (Miller, 2007; Fromhage et al., 2008; Foellmer, 2008). Trivers (1972) highlighted the fact that sex-specific mortality patterns coevolve with sex-specific patterns of parental investment. Sex role models that explicitly derive the relationship between the OSR and ASR are thus long overdue.

\subsection{When many factors interact you need mathematical models}

Having considered several factors that we believe are important for sex role evolution in most taxa, we need to consider how they interact. It is, however, almost impossible to predict the outcome of multiple interactions using only verbal arguments. We therefore require a mathematical model that integrates the preceding arguments into a general framework. Our aim is to explain from first principles the causal routes that can lead to one sex caring more than the other. Our model is designed to provide a broad foundation upon which future models can be built for investigating more restrictive, taxon-specific scenarios (e.g. filial cannibalism that occurs during parental care in fish) or for including additional factors, such as specific life history correlations between past investment and current body condition, or a trade-off between parental investment and certainty of paternity (e.g. mediated by investment in sperm competition: Lorch, 2002).

The most important features of our model are: (a) each sex can adjust how much it invests in parental care; (b) it does not assume that the OSR predicts competition, instead we calculate the average fitness return an individual male or female gains per unit time when competing for mates or caring; (c) we can monitor the feedback between the OSR, the ASR and how much parental care each sex provides. This feedback takes into account the Fisher condition, permits the evolution of sex-specific life histories, and highlights the distinction between mortality caused by mating competition and by parenting activities (e.g. Liker \& Székely, 2005). The model can be used to investigate the extent to which sex roles depend on: (i) the care needs of offspring, (ii) sexual selection, (iii) multiple mating (which influences expected parentage), (iv) the OSR and (v) the ASR.

\section{The model basics}

For all terminology the superscript $\sim$ represents male parameters. As a simple mnemonic we note that it resembles the tail of a sperm.

\subsection{Mating and expected share of parentage}

Offspring are produced when a group with an average of $n$ females and $\tilde{n}$ males mate with each other. This simple 
book-keeping device can represent a wide range of breeding systems (e.g. monandry, polyandry, haremdefence, extra-pair paternity, group spawning, eggdumping and communal nesting). If $n=\tilde{n}=1$ there is strict monogamy. If $n=1$ and $\tilde{n}>1$ then females either mate multiply (including extra-pair mating) or a group of males are spawning with a single female. If both $n$ and $\tilde{n}$ are high then there is both communal spawning and mixed paternity as occurs in, say, communally breeding frogs with sperm competition that share nest sites (e.g. Jennions et al., 1992). We make the simplifying assumption, readily modified in future models, that parentage is, on average, shared evenly within the group (i.e. $1 / n$ and $1 / \tilde{n})$. Phenotypic variation among individuals of each sex therefore has no effect on their share of parentage (e.g. due to differential female fecundity in communal egglayers, or variation among males in sperm competitiveness). Likewise, we exclude the possibility that otherwise identical males adjust their ejaculates in the light of information about the timing of mating or the status of their mate to elevate their share of paternity (e.g. no information on sperm competition roles or female status; for models see Parker, 1990; Ball \& Parker, 2007).

The parameters $n$ and $\tilde{n}$ determine expected parentage. This is important because parents should not waste parental investment on offspring to whom they are unrelated. In a given mating a male might not sire young but, on average, each male will sire $1 / \tilde{n}$ of the offspring produced. In contrast, a female can typically be certain about how many young she contributes to a brood, even if several females contribute to the brood $(n>1)$. Even so, her expected share of parentage in the whole brood still equals $1 / n$ so discounting total offspring production (per mating event) by this factor ensures that her fitness is correctly calculated. For identical values of $n$ and $\tilde{n}$, variance in male parentage will be greater than that of females but the mean values are the same. This allows us to analyse expected fitness gain by both sexes within the framework provided in Fig. 1. In nature, however, the actual parameter values often differ between the sexes (typically $\tilde{n}>n$ ), so most of our analyses will be based on sex-specificity of the curves and lines depicted in Fig. 1.

\subsection{Time is the currency}

After eggs are fertilized, parents stay with the current offspring for a time period $T$ or $\tilde{T}$ until they abandon them to return to the mating pool (Fig. 2). Some earlier models have included a term to indicate a sex-specific interval during which gametes are replenished that occurs after parental care ends, but before a parent returns to the mating pool (e.g. Yamamura $\&$ Tsuji, 1993). To reduce the number of terms in our model without loss of generality, we simply assume that a deserting parent is immediately physically capable of mating. However, we can take into account that it takes longer to produce eggs than sperm such that females

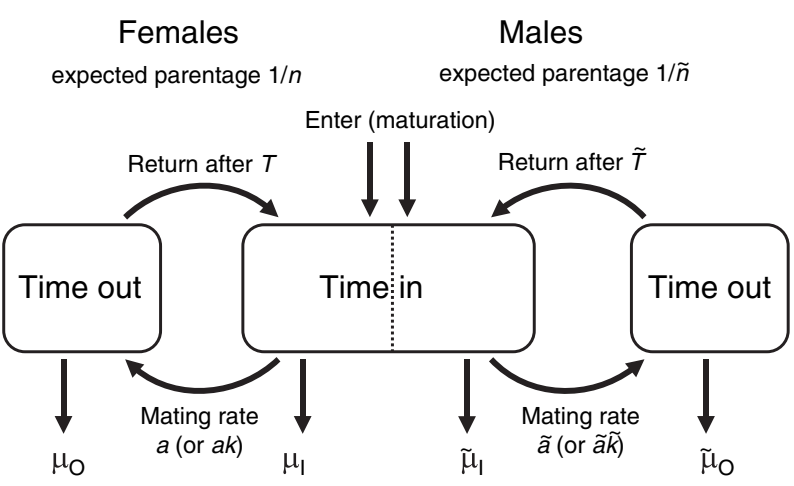

Fig. 2 A schematic to illustrate the use of a 'time in' and 'time out' approach to sex role evolution.

have a lower PRR by setting minima for $T$ and $\tilde{T}$ such that $T_{\text {min }}>\tilde{T}_{\text {min }}$. So, if parental investment remains minimal, females will take longer to re-enter the mating pool. In practice, however, the addition of minima (equivalent to assuming there is a post-care gamete replenishment interval) has no effect on our main findings.

In nature, the extent of parental care is a good predictor of offspring condition (e.g. Field $\delta$ Brace, 2004), survival (e.g. Gubernick \& Teferi, 2000; MacColl \& Hatchwell, 2003; Wright, 2006), dispersal or recruitment into the adult population (e.g. Ridley $\&$ Raihani, 2007), and its effect can extend to offspring breeding success (e.g. Royle et al., 2002). The returns for parents must, however, eventually diminish as the total amount of parental investment they provide increases (Fig. 1). This is illustrated by the observation that when care is experimentally terminated by removing a parent, the immediate effect this has on offspring survival is generally weaker for offspring who have received extended parental care, and are close to independence, than those who have received less care and are still small (e.g. Keenleyside \& Mackereth, 1992). In other words, for relatively independent offspring, a small amount of additional care improves their future prospects less than the same amount of additional care delivered earlier. The marginal benefit of additional care eventually declines which, in our model, means that delaying desertion time $T$ for too long no longer significantly improves offspring survival (Fig. 1).

\subsection{Sexual conflict over the duration of care}

A decision has to be made about how to combine the effects of each parent's care when there are two or more parents. Here, we simply assume that offspring fitness is a function of the sum of all parental care $\left(T_{\text {total }}=n T+\tilde{n} \tilde{T}\right)$. This approach immediately incorporates the notion of sexual conflict because individuals of both sexes would benefit if those of the other sex cared for longer. In our model, sexual conflict is played out over evolutionary 
time through shifts in the timing of desertion by each sex, rather than in the short-term through, say, behavioural adjustments in rates of provisioning in response to the immediate efforts of a breeding partner. We assume that two units of care by one parent are equivalent to one unit of biparental care. In the Conclusions section we will consider what happens if we relax this assumption. For now we simply note that if both sexes provide care of an identical quality this assumption will not generate biased sex roles.

In Fig. la we depict several possible functions $S\left(T_{\text {total }}\right)$ that relate offspring survival (or more generally, reproductive value) to total parental care. While our theoretical results apply for any shape of $S$, our current examples use an S-shaped function in which offspring survival is zero without parental care, followed by increasing returns until the marginal benefits begin to diminish (equations from Bulmer \& Parker, 2002). The function can be interpreted as the survival of a single offspring when brood size equals 1 , or the expected number of surviving offspring in a larger brood. When divided by $n$ or $\tilde{n}$ it can also be used to track the effects of lower expected parentage when there is mixed parentage (Fig. 1).

Figure 1 offers a simple graphic aid to focus attention on the relative costs and benefits of caring for and deserting offspring. A parent's expected parentage in a set of offspring will directly influence investment in care because it determines the average relatedness between a social parent and offspring. For a caregiver the maximum obtainable benefit is directly related to the proportion of offspring for whom it is a genetic parent. The proportion of offspring surviving should therefore be discounted by relatedness so that the maximum and marginal benefits of care decline when a parent has lower expected parentage (e.g. $25 \%, 50 \%$ or $100 \%$ of total offspring produced in Fig. la,b). A parent benefits from investing less in care ( $T$ or $\tilde{T}$ ) if the current marginal gain when staying with offspring is less than the expected fitness gained by deserting and re-entering the mating pool. Marginal gains are smaller for parents with lower expected parentage (Fig. la) so, for the same future pay-off (the horizontal line in Fig. 1b), a parent with low expected parentage will desert sooner. As $\tilde{n}$ or $n$ increases, male and female care, respectively, should decrease. As generally predicted, monogamy decreases sexual conflict over parental care.

For any given benefit of caring, parental care should be prolonged by parents with lower expected success once they return to the mating pool (lower elevation of the horizontal line showing the future pay-off, Fig. 1c). The future pay-off from desertion is directly proportional to how soon a parent can mate and leave the mating pool (i.e. parental mating rate). A useful model must take into account the frequency-dependence of each sexes mating rate that is created by the Fisher condition: individuals of the more common sex in the mating pool will reproduce less often. If all else is equal between the sexes (e.g. $n=\tilde{n})$, the sex towards which the OSR is currently biased is under selection to provide more care (see also Grafen $\&$ Sibly, 1978).

This interpretation of the outcome of a trade-off between caring and mate searching is, however, premature because it neglects a potentially confounding factor. Each mating group comprises $n+\tilde{n}$ individuals providing $n T+\tilde{n} \tilde{T}$ units of care. Offspring survival depends on the care decisions of all parents. A selfconsistent parental care model requires that the amount of care by each individual in a mating group affects the optimal level of care by every other member of the group (e.g. Houston \& McNamara, 2002, 2005; Fromhage et al., 2007). As already noted, however, care decisions are influenced by mate availability, which itself depends on how much care each sex provides. This creates a natural link between the theories of sexual conflict and parental investment (and argues against interpreting them as alternative hypotheses, Thomas \& Székely, 2005). A sensible model of sex role evolution must include reciprocal feedback between the OSR and how much care each sex provides.

\section{The full model}

We assume that individuals breed continuously in a large, stable population in groups of $n$ females and $\tilde{n}$ males that stay with offspring for $T$ and $\tilde{T}$ units of time, respectively, before deserting (Fig. 2). The average mating rate for individuals that seek mates is $a$ for females and $\tilde{a}$ for males, and we have ensured that mating rates are derived self-consistently so that the Fisher condition is satisfied (the details are provided in the Appendix). The variables $a$ and $\tilde{a}$ characterize mean mating rates, but we additionally need to consider variance in these rates because mating may not be random. Sexual selection requires variance in mating success that is not attributable to random effects but covaries with a specific trait (Shuster $\&$ Wade, 2003). This variance is important for sex role evolution as it elevates the mating rates of certain individuals above that of others. This will shift the balance between caring and competing for any individual who has mated (Fig. 1c). Accordingly, our model measures the variance in mating success through its effect on the predicted future success of individuals who are currently mated (in 'time out' state). The parameters $k$ and $\tilde{k}$ indicate the strength of sexual selection by elevating the expected mating rate to $a k$ (for females) or $\tilde{a} \tilde{k}$ (for males) for individuals who have mated (so that genes they carry that affect decisions about the timing of desertion are more often exposed to selection) (Queller, 1997).

If there is no variance in mating success, or it is only random variation, then a mated individual's status does not predict its future mating success. The future mating rate of a mated male or female is no different from a randomly chosen male or female in the mating pool when $\tilde{k}$ or $k=1$ respectively. When $k>1$ or $\tilde{k}>1$, 
mating individuals comprise a subclass of individuals with traits that generate above-average mating success. One interpretation is that categories are strict: some individuals belong to an elite class which comprises $1 / \tilde{k}$ of the males and $1 / k$ of the females, and only elite individuals mate (and, on average, they do so equally often). A second interpretation, when, say, the male parameter $\tilde{k}>1$, is that there is continuous variation in a male trait like body size that correlates with mating success. Mated males are therefore larger than average and their future mating rate is $\tilde{k}$ times that of the average male.

\subsection{The 'time in, time out' framework}

We assume continuous breeding which allows us to employ a variant of the familiar 'time in, time out' framework initiated by Grafen \& Sibly (1978) and developed by Clutton-Brock \& Parker (1992) (for other uses see Parker \& Simmons, 1996; Wiklund et al., 1998; Kokko \& Monaghan, 2001; Kokko \& Wong, 2007). Individuals are either 'in' and available to mate or 'out' and unable to do so because they are providing care (Fig. 2). In the original model of Clutton-Brock \& Parker (1992), for a fixed ASR, the OSR is simply proportional to (male time in/female time in). In our model time out is denoted $T$ or $\tilde{T}$. Unlike their model, however, we allow the duration of time out and the breeding cycle for each sex to evolve. If we had fixed these parameters, then we could calculate 'time in' as 'breeding cycle duration time out'. We did not do this because the critical feature of our model is that $T$ and $\tilde{T}$ evolve in response to changes in other parameters (including each other). This means that the OSR will also evolve. We note that the sex ratio at maturation is equivalent to the primary sex ratio $r$ if there is no bias in juvenile mortality. Females and males can die at any time with instantaneous mortality rates of $\mu_{\mathrm{I}}$ and $\tilde{\mu}_{\mathrm{I}}$ while competing for mates (time in) and $\mu_{\mathrm{O}}$ and $\tilde{\mu}_{\mathrm{O}}$ while caring (time out) (Fig. 2). Given a stable population we can then evaluate fitness over infinitesimal time intervals using the methods of Härdling et al. (2003) so that all variables are evaluated for the current sex ratios.

To summarize, our model identifies the equilibrium values of $T$ and $\tilde{T}$ for a given combination of values of five fixed parameters $r, n, k, \mu_{\mathrm{I}}$ and $\mu_{\mathrm{O}}$ (and the male equivalents) while mating rates $a$ are self-consistently derived. The scenarios we consider include cases where the primary sex ratio $r$ (which determines the sex ratio at maturity) differs from $1: 1$, as well as cases in which $r=1$ but ASRs are biased because the sexes specialize in activities that carry different risks. This contrast allows us to test what effect the ASR has on parental care separately from any effect of mortality rates (which influence sex role evolution directly, by changing the relative risk of caring or competing, and indirectly by changing sex ratios).

\subsection{Solving the model}

To solve the model we must quantify the marginal gains argument in Fig. 1 for a focal individual, while simultaneously taking into account how much care is provided by others. We begin by considering the options for an individual, say a male, who either deserts his offspring now to seek a new mate or stays with them for an infinitesimal unit of time longer before departing. If he behaves like the average male and stops providing care after time $\tilde{T}$ has elapsed, his offspring will survive with probability $S=S(n T+\tilde{n} \tilde{T})$. His instantaneous rate of fitness gain (the marginal benefit) is:

$$
\frac{\tilde{a} \tilde{k}}{\tilde{n}} S-\tilde{\mu}_{1}
$$

Here, $\tilde{a} \tilde{k}$ is the probability that a male who returns to the mating pool finds a mate during an infinitesimal unit of time. $S$ is divided by $\tilde{n}$ to account for a male's expected paternity. The benefit of desertion (eqn 1) can be contrasted with the gain accrued if the male continues to care for the current brood for an infinitesimal unit of time longer:

$$
\frac{S^{\prime}}{\tilde{n}}-\tilde{\mu}_{\mathrm{O}}
$$

In these expressions, offspring survival $S$ is evaluated at current care levels, $S=S(n T+\tilde{n} \tilde{T})$ and $S^{\prime}$ is the marginal benefit in terms of offspring survival when the total care received increases. For the example depicted in Fig. 1a, we use $S\left(T_{\text {total }}\right)=\exp \left(-\alpha / T_{\text {total }}\right)$, which implies $S^{\prime}=\alpha \exp \left(-\alpha / T_{\text {total }}\right) / T_{\text {total }}{ }^{2}$.

The reason we can combine the mixed currencies of offspring survival $(S)$ and parental survival $(\mu)$ in (1) and (2) is by assuming that offspring enter the 'time in' state immediately after independence so that they are as valuable as a parent in the 'time in' state. If we relax this assumption by assuming that juvenile mortality occurs after independence this is equivalent to changing the shape of $S$ (e.g. the maximum proportion of offspring surviving is $<1$, Fig. la). This does not affect our conclusions about sex roles.

The selection differential for care is expression (2) minus expression (1). This simplifies to:

$$
\frac{S^{\prime}-S \tilde{a} \tilde{k}}{\tilde{n}}+\tilde{\mu}_{\mathrm{I}}-\tilde{\mu}_{\mathrm{O}}
$$

The average male mating rate that satisfies the Fisher condition is $\tilde{a}=M r_{\mathrm{O}}{ }^{-1 / 2} \tilde{n}$ (see Appendix), where $r_{\mathrm{O}}$ is the OSR (males : females) and $M$ is a species-specific parameter that describes how often individuals encounter one another. After substitution of $\tilde{a}$ and simplification of the selection differential, the requirement for prolonging male care is:

$$
\frac{S^{\prime}}{\tilde{n}}-S M \tilde{k} r_{\mathrm{O}}^{-1 / 2}+\tilde{\mu}_{\mathrm{I}}-\tilde{\mu}_{\mathrm{O}}>0
$$


A similar derivation yields, for females,

$$
\frac{S^{\prime}}{n}-S M k r_{\mathrm{O}}^{1 / 2}+\mu_{\mathrm{I}}-\mu_{\mathrm{O}}>0
$$

The power to which $r_{\mathrm{O}}$ is raised differs for males and females. If the OSR is male-biased $\left(r_{\mathrm{O}}>1\right)$, then females in the mating pool mate sooner than males. The terms $S M \tilde{k} r_{\mathrm{O}}{ }^{-1 / 2}$ and $S M k r_{\mathrm{O}}{ }^{1 / 2}$ can be considered the mating opportunity cost of staying with the current brood for males and females respectively.

\section{Model results}

\subsection{The first four key conclusions}

To start, consider the simplest scenario where mortality rates are the same whether caring or mate searching and are identical for both sexes. This reduces inequalities 3(a) and 3 (b) to:

$$
\frac{S^{\prime}}{\tilde{n}}>S M \tilde{k} r_{\mathrm{O}}^{-1 / 2}
$$

and

$$
\frac{S^{\prime}}{n}>S M k r_{\mathrm{O}}^{1 / 2}
$$

There are only three terms that differ between the sexes: $\tilde{n}$ or $n, \tilde{k}$ or $k$ and $r_{\mathrm{O}}^{-1 / 2}$ or $r_{\mathrm{O}}{ }^{1 / 2}$. This leads to four general results:

Result 1. Low levels of care, high marginal gains and low mate encounter rates select for more care. There is selection on both sexes to invest more in care $(\tilde{T}$ or $T)$ when total parental investment is low so that $S$ is small, likewise care is selected for if offspring benefit greatly from additional care so that $S^{\prime}$ is large. The opportunity cost of caring is also greater when the absolute mate encounter rate $M$ is higher.

Result 2. Sexual selection reduces care. If there is sexual selection on males $(\tilde{k}>1$ so that some males can consistently mate sooner than others), males are selected to care less. The equivalent argument holds true for females (when $k>1$ ). All else being equal, whichever sex is subject to stronger sexual selection (i.e. has higher non-random variance in mating success) will provide less care.

Result 3. Low parentage reduces care. If females mate multiply $(\tilde{n}>1)$ males are selected to provide less care. Similarly, if females breed communally so that they are uncertain of the parentage of specific offspring $(n>1)$ they are also selected to provide less care. All else being equal, females will provide more care if $\tilde{n}>n$.

Result 4. The direct role of the OSR is to prevent sex roles from diverging. A male-biased OSR reduces the relative mating opportunity cost for males because $r_{\mathrm{O}}{ }^{-1 / 2}<r_{\mathrm{O}}{ }^{1 / 2}$ when $r_{\mathrm{O}}>1$. Thus, when the OSR is male-biased, males are selected to provide more care than females. When the OSR is female biased the reverse is true. The OSR is partly determined by the relative amount of parental care each sex provides (see Appendix) so the net effect is that whichever sex currently cares less will be selected to care more.

\subsubsection{Evaluation of the first four results}

The first three results are relatively unremarkable. We will not comment further on the first. The second result is also unsurprising. It agrees with the simple model of Queller (1997), the verbal argument of Trivers (1972) and formal models that build on these studies. However, it is worth reiterating that this general conclusion might be reversed if females prefer good fathers so that male parental investment is a sexually selected trait (Hoelzer, 1989). It is also important to note that our model excludes males adjusting the level of care they provide in response to their own assessment of their future mating prospects. As our model predicts a single value for male care, it excludes adaptive reaction norms, which would require selection on males who perceive themselves to be more (or less) successful than other mated males to provide less (or more) care. This perception could, for example, be based on continuous variation in a sexually selected trait that influences male attractiveness. Instead, our model assumes that selection is based on the average mating success of mated males. Even if males did evolve to differentially allocate parental care, however, the average level of care should still diminish as average paternity decreases.

Intriguingly, accounts of sex role evolution virtually always mention that sexual selection reduces male care (result 2), but the consequences of sperm competition (result 3) are given far less credit in driving sex role divergence. This is unfortunate as sperm competition is taxonomically widespread and result 3 applies whenever multiple mating or external fertilization creates mixed paternity, irrespective of the reason why multiple mating evolves in the first place. For example, it can evolve because polyandrous females increase the total male parental care that a brood receives (e.g. Harada $\&$ Iwasa, 1996; Houston et al., 1997). Such a direct benefit of multiple mating is readily compatible with result 3, which refers only to the per capita male contribution to care declining with multiple mating.

In our view, the fourth result is the most striking and yet the most poorly appreciated. This counterintuitive result illustrates why most verbal accounts of sex role evolution are unsatisfactory: they fail to correctly take into account the Fisher condition. Result 4 also raises an intriguing empirical question because of its potential tension with result 2 . When the OSR is male-biased, we expect intense sexual selection because of competition for mates (Trivers, 1972). This increases the temptation for some males to desert if they are consistently better competitors than others (result 2), but how does this compare to the level of counter-selection on males to invest more in care overall (result 4)? There is a 
fascinating feedback at work here because if the net outcome is greater male investment in care this will, in turn, reduce the male bias in the OSR, which might weaken sexual selection on males.

\subsection{What determines the intensity of sexual selection?}

\subsubsection{Sexual selection and the OSR}

We noted earlier that most theoreticians, and numerous fieldworkers, simply assume that a male-biased OSR will increase sexual selection on males. They then assume that this suffices to explain sex role divergence. This is, for example, integral to Trivers' original formulation for the evolution of sex roles, and was codified by Emlen 8 Oring (1977) who classified mating systems based on the OSR. However, the potential conflict between results 2 and 4 shows that the precise relationship between sexual selection and the OSR is critical. If sexual selection does not intensify sufficiently quickly as the OSR changes, then sex roles will diverge little because, by itself, a biased OSR hinders divergence in parental investment.

There are many possible measures of sexual selection (non-random variance), but in the context of parental investment evolution, the relevant measure is how much it elevates the future expected mating rate of individuals who have mated (result 2). Therefore, to quantify the relationship between sexual selection and the OSR in our model we would have to specify $\tilde{k}$ as a function of $r_{\mathrm{O}}$. There are, however, many possible relationships that depend on specific biological assumptions. Unlike the case for male and female mating rates, there is no constraint that forces this relationship to take a specific form. There are situations in which there is no relationship (e.g. no sexual selection on males for the OSR range in which there is sperm limitation; Levitan, 1996), or even a negative relationship between the intensity of sexual selection on males and the OSR (e.g. Mills $\delta$ Reynolds, 2003; see Section 3.1.). The prevailing population density could also greatly modify the relationship (for a review see Kokko \& Rankin, 2006). Empirical answers are required. It would be useful to know whether variation among taxa in the relationship between $\tilde{k}$ or $k$ and the OSR predicts sex role variation across species.

Obviously, once sex differences evolve, selection can become sex-specific in numerous qualitative as well as quantitative ways (Clutton-Brock, 2007). In our general context, however, it is parsimonious to assume that $\tilde{k}$ and $k$ have identical relationships with the OSR (aside from being mirror-images) because we are considering the evolution of sex roles from an ancestral condition where the sexes only differ in gamete production. It would, however, be interesting to calculate the relationships between $\tilde{k}, k$ and $r_{\mathrm{O}}$ within extant species to test for sexual asymmetries. For example, does an OSR of $2: 1$ generate stronger sexual selection on males than a $1: 2$ OSR generates on females?

\subsubsection{Sexual selection and the coevolution of other traits} In the longer term $\tilde{k}$ might coevolve with parameters that are currently fixed in our model. For example, $\tilde{k}$ might coevolve with the mortality associated with mating competition $\tilde{\mu}_{\mathrm{I}}$. Traits, like larger size or weapons, that enhance male mating success often evolve under the constraint of a trade-off between mating rate, $\tilde{a} \tilde{k}$, and mortality during mating competition, $\tilde{\mu}_{\mathrm{I}}$. This basic tradeoff can take several forms though, so specific predictions vary depending on life histories. Sexual dimorphism resulting from sexual selection can also lead to sexspecific juvenile mortality rates (e.g. Clutton-Brock et al., 1985). In our model this would alter the sex ratio at maturation rather than $\tilde{\mu}_{\mathrm{I}}$, and necessitate sex-specific terms for juvenile survival that are a function of $\tilde{k}$ and $k$. The intensity of sexual selection on males might also have an age-dependent effect on $\tilde{\mu}_{\mathrm{I}}$ because there is evidence that males in polygynous species senesce faster than females (Clutton-Brock \& Isvaran, 2007). Modelling the relationship between any male response to sexual selection and $\tilde{k}$ is a challenge. $\tilde{k}$ is not an inherent property of an individual but a measure of variance in male mating success. So, for example, when all males evolve to be larger or to invest more in weapons this need not change the variance in mating success.

To keep the current model as simple and general as possible we do not address the complications of these forms of coevolution. In future, however, adding explicit trade-offs could identify values of $\tilde{k}$ that are more likely to correspond to a 'possible state of the world' (sensu Houston et al., 2005). At present, our model does not predict a value for $\tilde{k}$. We simply assign a value and quantify the effect on sex roles. It is noteworthy that high values of $\tilde{k}$ can counteract but never remove opposing selection on males to pursue more profitable fitnessenhancing options than mating competition when the OSR is male-biased. The results we have derived explore a single option: to provide more parental care. Other options include increased investment in ensuring paternity, sperm competitiveness (e.g. Lorch, 2002) or somatic growth if this increases future mating success or fecundity (e.g. Sargent \& Gross, 1993).

\subsection{Sex role divergence in models with continuous or binary decisions}

Many previous studies of parental care treated caring or desertion as a binary decision (e.g. Yamamura $\delta$ Tsuji, 1993; Webb et al., 1999). These models illuminate why sex role divergence is often incomplete: as deserters become more common, their mating success decreases, until deserting and caring are equally profitable. In settings that only permit a binary choice between caring and deserting, incomplete sex role divergence can only 
mean that some individuals of a given sex desert while others care. There are species with clearly defined alternative strategies where only some males care (for reviews see Gross, 1996; Taborsky, 2001; Mank \& Avise, 2006; Reichard et al., 2007; Tomkins \& Hazel, 2007), but the level of care vary along a continuum in most species.

Binary-choice models identify the proportion of caring and non-caring males and females that will generate sex roles and associated sex-specific mating opportunities that yield a stable equilibrium. This state could, however, also be reached if a certain amount of care per sex was divided more evenly among all same-sex carers, i.e. by treating care as a continuous variable. Our model falls into this category by letting the time spent caring evolve: sex roles diverge completely if the evolutionary end point is no investment (beyond the minimal time out required to replenish gametes) by one sex.

\subsection{What determines whether sex roles do or do not diverge?}

In Fig. 3 we provide examples of evolutionary trajectories for each sexes investment in care $(\tilde{T}$ or $T)$. These examples confirm that sex roles do not always diverge. When mating is random $(\tilde{k}=k=1)$ and there is no multiple mating $(\tilde{n}=n=1)$, negatively frequencydependent selection results in the evolution of egalitarian sex roles (Fig. 3a). When females mate multiply $(\tilde{n}>1$, $n=1$ ), female-biased care evolves (Fig. $3 b$ ). This is not due to non-random mating or fertilization success (e.g. some males being more successful at gaining extra-pair copulations or stronger at sperm competition) as we explicitly excluded these processes $(\tilde{k}=k=1)$. It only arises because the value of male care diminishes with lower expected paternity.

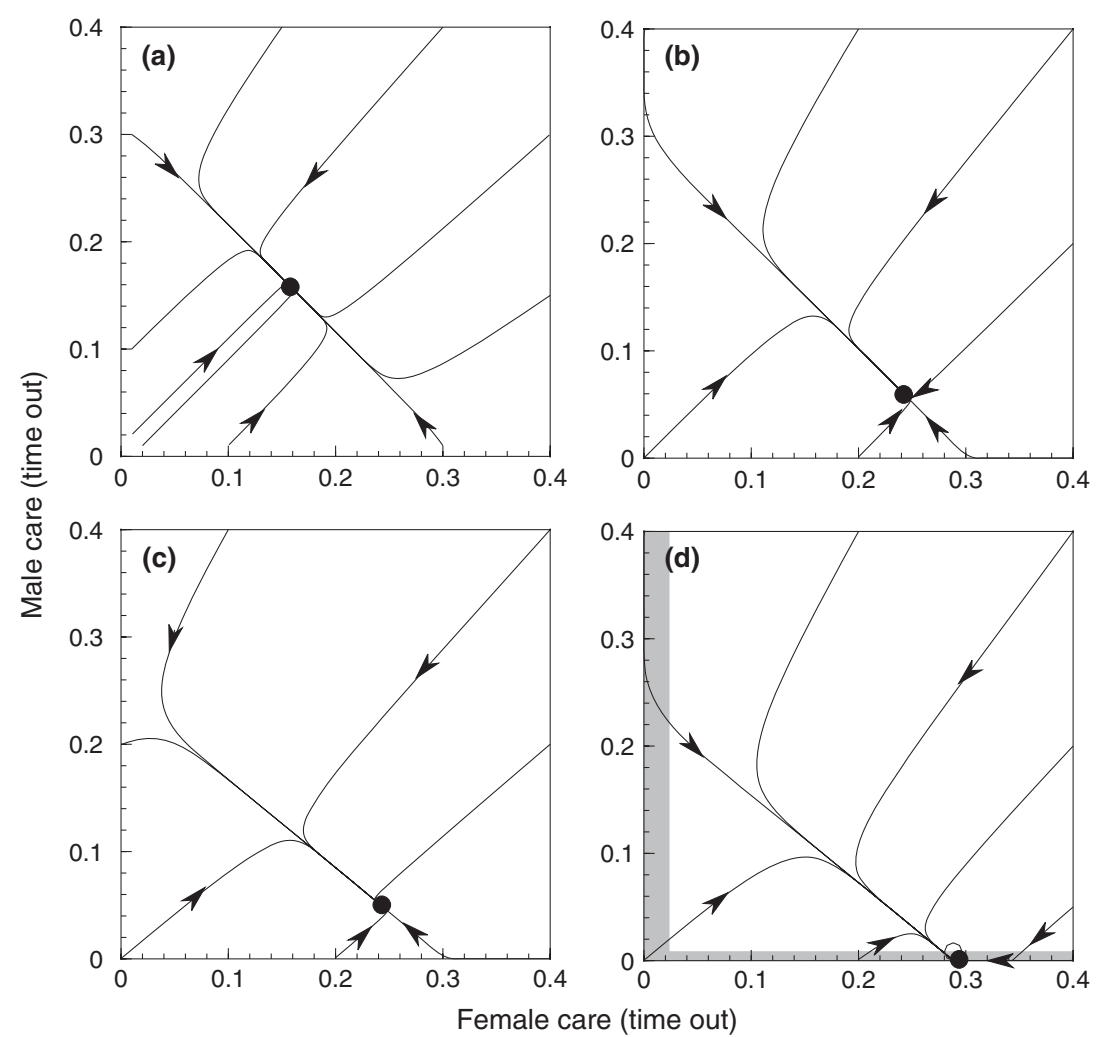

Fig. 3 Evolutionary trajectories assuming that the duration of care by each sex evolves at a rate proportional to the sex-specific selection differentials in eqn (3) (see text). Offspring survival follows $S\left(T_{\text {total }}\right)=\exp \left(-0.1 / T_{\text {total }}\right)$ and there are no biases in mortality $\left(\tilde{\mu}_{\mathrm{I}}=\mu_{\mathrm{I}}=\tilde{\mu}_{\mathrm{O}}=\right.$ $\left.\mu_{\mathrm{O}}=0.01\right)$, the primary sex ratio is even $(r=1)$, and individuals encounter on average one mate per time unit $(M=1)$. We assume no genetic covariance in 'time out' between the sexes or any sexual differences in the amount of genetic variation in the trait. (a), $k=\tilde{k}=1, n=\tilde{n}=1$. Regardless of the ancestral state (i.e. where evolution starts), the end point is egalitarian care (e.g. even with an initial asymmetry where females invest far more than males). (b) Similar to (a) but with weak sexual selection on males, $\tilde{k}=1.2$. Biparental care evolves with females providing more care than males. (c) Similar to (a) but females mate with an average of $\tilde{n}=1.2$ males. The result is again biparental care with females providing more care than males. (d) Sexual selection in combination with multiple mating, $\tilde{k}=\tilde{n}=1.2$. The result is female-only care, and male parental investment evolves to the lowest value possible. This is either zero (black dot) or a slightly larger value (open dot). This larger value takes into account that there is a minimum parental investment in the form of gamete production for each sex, which is greater for females than males, as indicated by the grey zones (see text for details). 
A similar degree of female-biased care evolves if sexual selection is stronger on males than on females $(\tilde{k}>k)$ when there is complete certainty of parentage for both sexes ('serial monogamy', $\tilde{n}=n=1$ ) (Fig. 3c). If we increase the value of either $\tilde{k}$ or $\tilde{n}$ sufficiently in Fig. $3 \mathrm{~b}, \mathrm{c}$ this eventually leads to female-only care (not shown). Alternatively, complete sex role divergence will occur when moderately strong sexual selection on males is combined with a moderate reduction in expected paternity $(\tilde{k}=\tilde{n}=1.2$, Fig. $3 \mathrm{~d})$, even though neither factor on its own would lead to complete sex role divergence (Fig. 3b,c). In Fig. 3d we additionally illustrate the effect of having a lower boundary for 'time out' durations (e.g. $T_{\min }$ ): the width of the shaded areas differs for males and females to allow for a difference in the rate of gamete replenishment after mating. It is clear that this modification has a very minor effect on the model equilibria (the trajectories entering grey 'no-go' zones simply move along the boundaries of these zones). Indeed, there is no effect whenever care durations evolve to greatly exceed these lower boundaries (e.g. Fig. 3a-c).

\subsubsection{Past investment does not predict sex roles; multiple mating and sexual selection do}

Arguably the most important message from Fig. 3 is that the equilibrium does not depend on an initial bias towards greater female or male parental investment. The same equilibrium can be reached from all starting points (ancestral parental investment patterns). In Fig. 3a, for example, males and females provide equal care at equilibrium even when females initially provide more care than males, i.e. from all starting points below the diagonal line of equality. When the only sexual asymmetry is that of anisogamy, sex roles do not diverge. The equilibria reached in Fig. 3b-d reflect processes that, while they might be promoted by anisogamy, do not depend on its existence. One can, for example, conceive of an isogamous species in which sexual selection is greater on one sex, or there is a sex-specific mean number of individuals participating in a mating event.

That sexual selection and female multiple mating lead to greater female than male care (Fig. 3b-d) confirms the logic of Queller (1997). His argument holds even if there is a very large initial asymmetry in parental investment. Taken together, our findings refute any suggestion that females are directly selected to provide more care for their offspring simply because of their higher initial parental investment in the form of gametes (e.g. Trivers, 1972). This supports our own verbal argument (Section 2.2.) that anisogamy on its own does not lead to divergent sex roles.

An astute reader might be concerned that in the preceding argument we treat male care (in our model 'time out') and pre-mating parental investment (gamete production costs) as near synonymous. To do so, our interpretation of the labels of the axes in Fig. 3 has changed from 'care' to 'parental investment' (including that into gametes). This is legitimate: if we label the axes as 'parental investment' then gametic investment per mating simply creates a minimal investment for each axis (in Fig. $3 d$ the shaded area can now be interpreted as parental investment in gametes per mating). When the direction of evolution is away from both axes this has no effect on the equilibrium value (Fig. $3 a-c)$. If the equilibrium value is on the $x$-axis (i.e. female-only care), this simply means that the equilibrium point is at the minimum male investment value, rather than zero (i.e. the difference between open and filled dots in Fig. 3d).

\subsection{How to incorporate arguments about the ASR}

The case studies in Fig. 3 highlight several important principles, but they assume that adult mortalities are unaffected by the extent to which each sex spends time caring or competing. There is no interaction between sex roles and sex-specific life histories that could alter the ASR. This raises a question: what happens when the ASR is biased?

\subsubsection{Bias in the primary sex ratio}

The most fundamental reason for a biased ASR is a skewed primary sex ratio, $r$. There are adaptive reasons for individual parents to bias their offspring sex ratio (Frank, 1990), but this can occur in either direction. Although an overall bias in the primary sex ratio is possible, it tends to be small or absent, except in special cases such as haplodiploids (Uller et al., 2007). A biased ASR is more often a result of sex-specific mortality rates. Nonetheless, if we change $r$ this provides an insight into the effect of the ASR without having to simultaneously consider other ramifications of sex-specific mortalities. Change in $r$ is reflected in the sex ratio at maturation if juvenile mortality is not sex-specific. This allows us to focus solely on the effect of different numbers of adult females and males. It also means that we can use eqn (4), which is simpler than eqns (3a) and (3b). For now, we simply ignore another type of Fisherian frequencydependence: sex biases in $r$ can make parents value broods differently depending on the brood's sex ratio (Lessells, 1998).

When the sexes have the same mortality rates the ASR is synonymous with $r$ (Fig. 4). The ASR covaries with the OSR, but evolving parental investment patterns can either amplify or diminish the OSR bias from the baseline initially created by the ASR (Fig. 4a). When the ASR is biased, the Fisher condition makes it more profitable for the rarer sex to desert sooner. It follows that if the only difference between the sexes is anisogamy and the ASR is biased, there is frequency-dependent selection for increased parental investment by the more common sex and for less investment by the rarer sex. This continues until the OSR reaches $1: 1$ (lowest horizontal line in Fig. 4a). If the ASR is strongly biased, this process is 

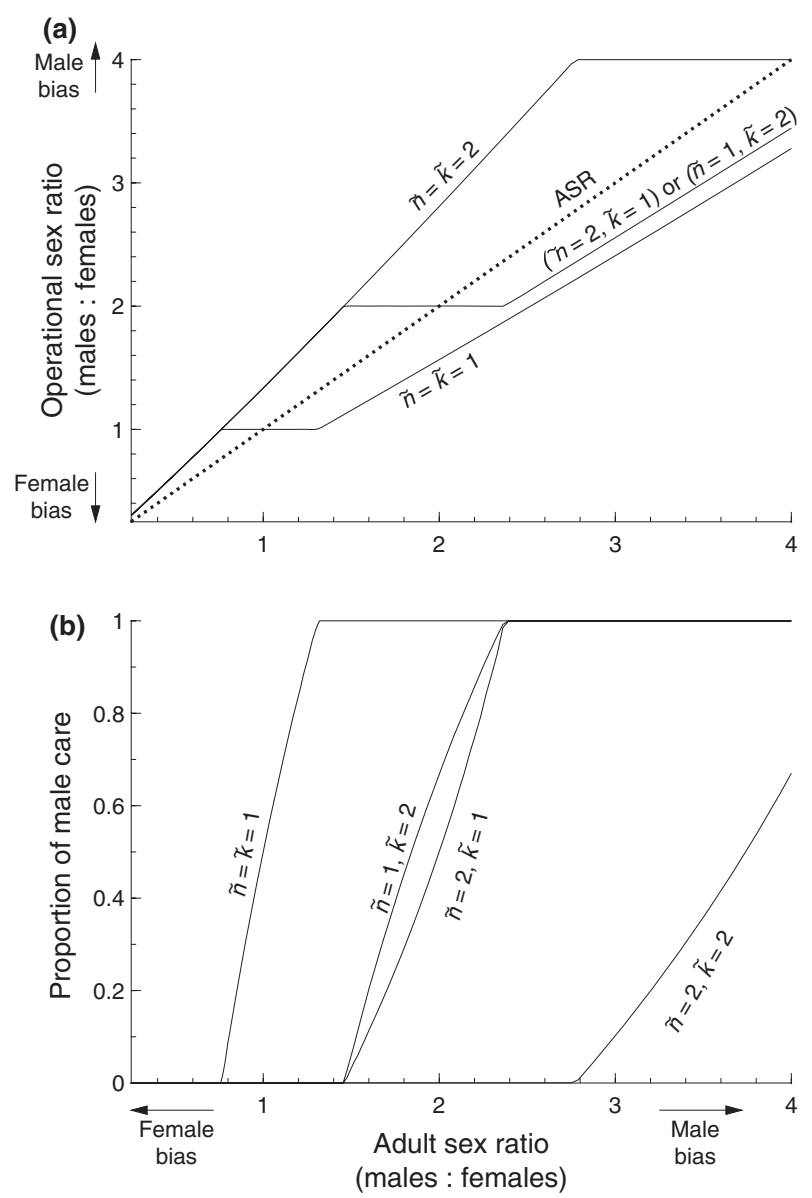

Fig. 4 The effect of adult sex ratios on (a) the operational sex ratio (OSR) and (b) the relative proportion of care provided by males, expressed as $\tilde{T} /(T+\tilde{T})$, at evolutionary equilibrium when the adult sex ratio (ASR) is biased due to a biased primary sex ratio $r$ (range $0.25 \ldots 4)$. For ease of reference a dotted line indicates the case where the ASR $=$ OSR. Mortalities are unbiased, $\tilde{\mu}_{\mathrm{I}}=\mu_{\mathrm{I}}=\tilde{\mu}_{\mathrm{O}}=\mu_{\mathrm{O}}=0.01$, $M=n=k=1$, offspring survival is as in Fig. 3, and there are four combinations of male parameters $\tilde{n}$ and $\tilde{k}$ which are indicated on the figures. With an extremely female-biased ASR there is female-only care, with an extremely male-biased ASR there is male-only care, and for intermediate ASR values populations evolve sex roles so that the OSR approaches values 1, 2 or 4 depending on $\tilde{n}$ and $\tilde{k}$ (note that the product $\tilde{n} \tilde{k}$ equals 1,2 or 4 in the different examples). The sex roles that satisfy eqn (3) in the $\tilde{n}=1, \tilde{k}=2$ case differ slightly from that in the $\tilde{n}=2, \tilde{k}=1$ case because $\tilde{n}$ influences the number and $\tilde{k}$ only the identity of mating males, so $\tilde{n}$ has a more direct influence on the OSR (see Appendix).

halted before an equal OSR is reached. Once the rarer sex no longer provides any parental care it cannot re-enter the mating pool any sooner. In this case the OSR evolves to be as close to equality as possible given the ASR (case $\tilde{n}=\tilde{k}=1$ in Fig. 4). Regardless of whether the ASR is female- or male-biased, the OSR is less biased than the ASR due to increased care by the more common sex and a corresponding decrease by the rarer sex.
7.5.2. The equilibrium OSR differs from $1: 1$ if the pay-offs of ongoing caring are sex-specific

The prediction that a strongly male-biased ASR selects for male care is robust to the presence of other factors that reduce male care (e.g. sexual selection $\tilde{k}>1$ and/or low expected parentage, $\tilde{n}>1)$. In these cases, the ASR bias necessary to reach a specific level of male care is simply increased (Fig. 4b). Again, the equilibrium level of male and female care is unaffected by any initial asymmetry in parental investment. Of course, if there is a difference in the benefits of caring between the sexes (e.g. females mate multiply, so that $\tilde{n}>1$ ), individuals of the sex with smaller gains provide less care. At the population level this results in an OSR that no longer evolves towards $1: 1$. For example, if $\tilde{n}=2$ or $\tilde{k}=2$ the equilibrium OSR is 2 , or as close to this value as possible when one sex no longer provides any care. If sexual selection acts in concert with lower expected paternity such that $\tilde{n}$ and $\tilde{k}$ both equal 2 , the corresponding 'target' OSR at which there is no selection for a change in parental investment patterns is 4 (Fig. 4a). The parental investment biases that lead to this OSR value are, however, a function of the ASR (Fig. 4b).

\subsubsection{Why does the ASR matter?}

The above scenarios reveal that the ASR can, by itself, affect the evolution of sex roles (see also Yamamura $\delta$ Tsuji, 1993; McNamara et al., 2000). This is not immediately apparent from inspection of eqns (3) or (4) that only have a term for the OSR. Why then does the ASR have such a strong effect? If both sexes care equally, the OSR bias will perfectly reflect any bias in the ASR. However, individuals of the rarer sex can always take advantage of the higher mating rate conferred by a biased OSR. They are therefore under selection to provide less care until sex roles have evolved so that this is no longer true. That happens either when the OSR has evolved to be even (Section 7.5.1.), or when it has reached an equilibrium value that differs from $1: 1$ for reasons described in Section 7.5.2. Thus the 'target' OSR is influenced by sex differences that affect expected parentage $(\tilde{n} \neq n)$ or the gains from deserting (i.e. sexual selection, $\tilde{k} \neq k)$. The magnitude of the shift in parental investment required to reach the 'target' OSR will depend on the starting point, which is the ASR. In sum, a biased ASR alters the sex roles that create an OSR at which the selection differentials for further sex role evolution (i.e. selection on $\tilde{T}$ or $T$ ) are zero.

\subsubsection{The OSR and ASR should not be conflated}

The tendency to equate the ASR and OSR is understandable given that the former has a strong effect on the latter (Clutton-Brock \& Parker, 1992; Kvarnemo \& Merilaita, 2006). Our findings emphasize, however, that while these ratios naturally covary when sex roles are fixed, their relationship becomes convoluted when sex roles evolve (see Appendix for details). In general, the OSR 
deviates from the ASR and shifts towards 1 if care duration is the only asymmetry between the sexes, and towards another value if there are other sex differences (Fig. 4a shows the OSR shifting towards 1, 2 and 4).

This result is important for empiricists. Knowing the ASR might account for differences among species or populations in the extent to which care is sex-biased even when the OSR is identical. Current emphasis on the OSR makes it hard to explain this type of variation. For example, the ASR can counter the direction in which we expect sex roles to evolve based on, say, a measure of the benefits of caring for each sex. Even if multiple paternity reduces the benefit males gain by caring, male-biased care might still evolve if the ASR is sufficiently male-biased that lost mating opportunity costs while caring are less than the gains derived by increasing offspring fitness.

\subsection{Adult mortality, the ASR and sex role divergence}

Although Fig. 4 is conceptually important, primary sex ratio biases are often small or absent in nature. Far more common are sex-specific mortality rates that must influence the ASR (mammals: Clutton-Brock et al., 1985; birds: Benito \& González-Solís, 2007). A common situation that we have so far ignored is that the ASR is biased because male and females have different life histories. This can easily happen once sex roles begin to diverge. We have already shown that the ASR influences the evolution of parental investment by each sex, so we must now consider the effect of this additional feedback loop.

To analyse it we need to consider sex- and stagespecific mortalities. If we look at eqns (3a) and (3b) there are two immediate processes that become clear. First, adult mortality rates directly affect the selection differential and select for more care if caring is safer than competing for mates. Second, and this will turn out surprisingly important, mortality rates have an indirect effect because they alter the OSR (via their direct effect on the ASR), and the OSR influences the selection differential for caring. These direct and indirect effects of mortality can change the direction of selection for care (see Appendix). We will now show that stage-specific mortality can lead to the evolution of both stronger and weaker divergence in sex roles.

\subsubsection{Self-reinforcing sex role divergence becomes more likely when competing for mates is riskier than parental care}

Consider a case where competing for mates is riskier than providing parental care $\left(\mu_{\mathrm{I}}>\mu_{\mathrm{O}}, \tilde{\mu}_{\mathrm{I}}>\tilde{\mu}_{\mathrm{O}}\right)$, but we assume no differences in the sex-specific ability to cope with these risks $\left(\mu_{\mathrm{I}}=\tilde{\mu}_{\mathrm{I}}, \mu_{\mathrm{O}}=\tilde{\mu}_{\mathrm{O}}\right)$. The earlier deserting sex becomes rarer because it spends more time risking death. Simultaneously, however, the Fisher condition favours desertion by the rare sex. It is tempting to ignore the feedback component of selection (the Fisher condition) and simply state that individuals should compete less when this is more dangerous. However, our model shows that feedback with sex roles can become an overriding factor.

Both sexes are obviously selected to avoid dangerous activities, but if females avoid the dangerous 'time in' more often than males (initially perhaps for other reasons, e.g. if multiple mating favours female care), then the mating success of surviving males can increase more sharply than one would predict by considering the male side of the argument only. As a one-sided view, one would predict no change in the mean success of deserting males if males die more frequently and surviving males mate correspondingly more often. However, when the female response is taken into account, a higher number of surviving females combines with a smaller number of surviving males. This amplifies the ASR bias to the extent that the expected success of surviving deserter males must more than compensate for the risks, and the net effect is selection for earlier male desertion (also see point 55 in Box 1). The larger the asymmetry in caring, the higher the mating success of males in 'time in' mediated via a shift in the ASR, which creates a self-reinforcing loop.

For much of this review we have argued that selfreinforcing selection for increasingly divergent sex roles is not a self-evident outcome of sex role evolution. But when desertion creates rarity, and rarity in turn selects for desertion, we have a case where a self-reinforcing process actually occurs. It requires the specific condition of higher mortality for deserting than caring individuals.

\subsubsection{Sex roles diverge less when parental care is riskier than competing for mates}

The above outcome is reversed when parental care ('time out') is the riskier activity $\left(\mu_{\mathrm{I}}<\mu_{\mathrm{O}}, \tilde{\mu}_{\mathrm{I}}<\tilde{\mu}_{\mathrm{O}}\right)$ so that individuals of the first sex to desert spend more time in the relatively safe 'time in' state. This amplifies survival of the sex that is more common in the mating pool, so they will take even longer to find a mate. According to the Fisher condition, this selects for it to increase investment in care. Now, instead of self-reinforcing selection, we find the opposite: emerging sex ratio biases counter the evolution of divergent sex roles. Again, this is a counterintuitive result: when care is the riskier activity there is selection that makes both sexes care more. This finding is only evident if one explicitly considers population-level feedbacks.

\subsubsection{A worked example to show that first intuitions are deceiving}

The above scenarios are illustrated in a case study in Fig. 5 where a population is initiated with equal male and female care. For the first 10000 generations the only reason for an asymmetry in care is weak sexual selection on males $(\tilde{k}>1)$. Competing for mates is assumed to be as risky as providing parental care. Biparental care 

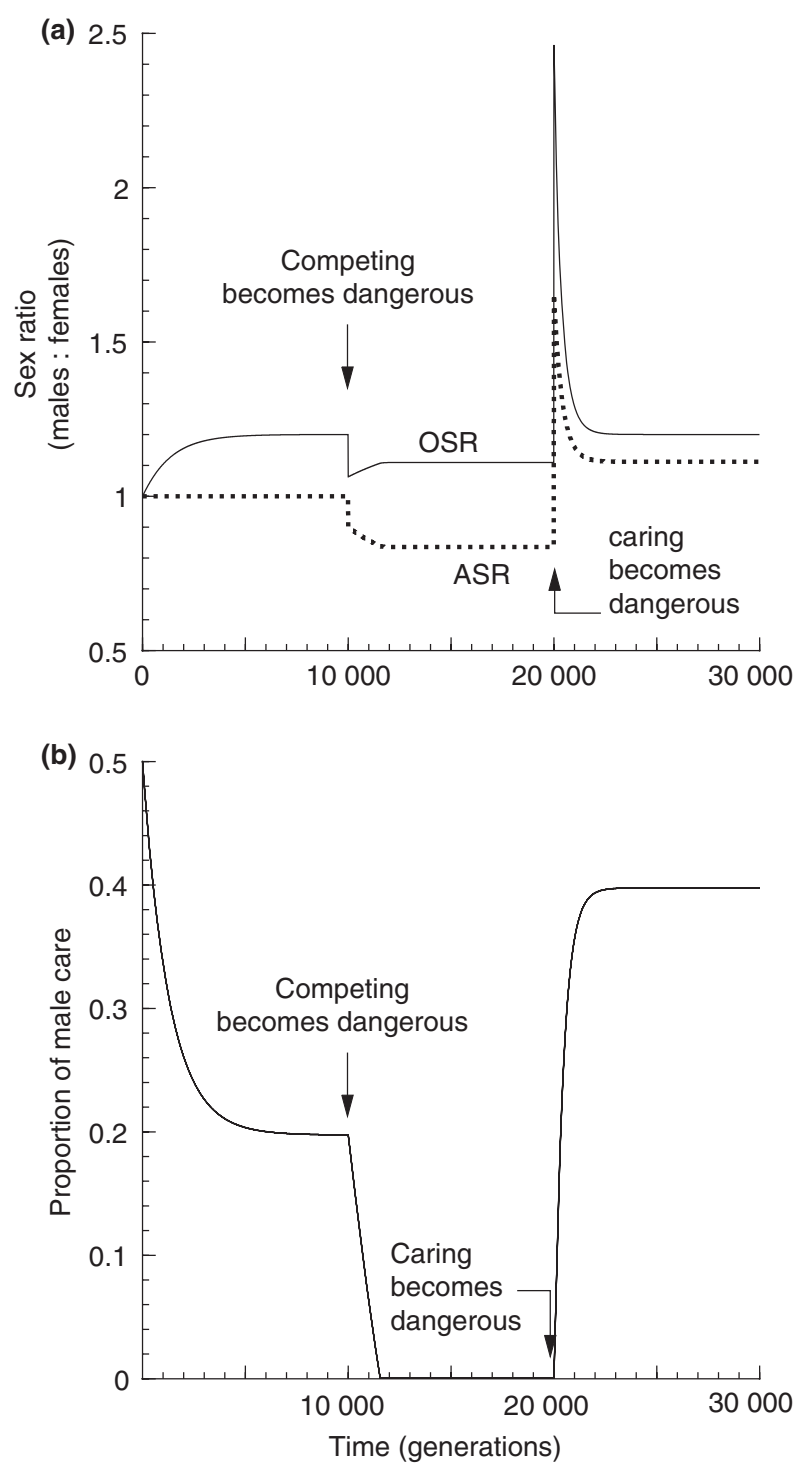

Fig. 5 Evolution over time in a population with three different mortality regimes. To ensure that transitional states are visible we assume that selection leads to a change in 'time out' traits at a rate $0.0001 \times$ the selection differential (eqn 3) per generation. Throughout we assume that offspring survival is as in Fig. 3, $M=n=\tilde{n}=k=1$, with mild sexual selection on males, $\tilde{k}=1.2$, and no genetic covariance in 'time out' between the sexes. For the first 10000 generations, $\tilde{\mu}_{\mathrm{I}}=\mu_{\mathrm{I}}=\tilde{\mu}_{\mathrm{O}}=\mu_{\mathrm{O}}=0.01$ (equal mortality), for the next $10000, \tilde{\mu}_{\mathrm{I}}=\mu_{\mathrm{I}}=0.03, \tilde{\mu}_{\mathrm{O}}=\mu_{\mathrm{O}}=0.01$ (competing is costly), and for the final 10000 generations $\tilde{\mu}_{\mathrm{O}}=\mu_{\mathrm{O}}=0.03$, $\tilde{\mu}_{\mathrm{I}}=\mu_{\mathrm{I}}=0.01$ (caring is costly). (a) Evolving values of the adult and operational sex ratio, (b) evolving proportion of care provided by males, expressed as $\tilde{T} /(T+\tilde{T})$.

evolves with males eventually providing $20 \%$ of care. This care asymmetry leads to a male-biased OSR, but the ASR is even because caring and competing are equally dangerous so the sexes have identical mortality rates. We then explore the effect of a sudden, externally imposed change in 'time in' mortality when mate searching at generation 10000 and another in 'time out' mortality when caring at generation 20000 .

At generation 10000 , competing for mates becomes three times more dangerous, per unit time, than caring for offspring. The immediate effect is a drop in the ASR: males lead riskier lives and the population consequently has more females than males. The OSR is still malebiased because females provide more care than males, but it is less male-biased than its 'target' value: the OSR at equilibrium should exceed 1 because $\tilde{k}>1$ (Sections 7.5.2.-7.5.3.). This predicts evolution towards a more male-biased OSR, i.e. less male care. While this mathematical explanation involves a fairly complicated process that makes the OSR reach a biased equilibrium from a given starting point, the biological insight is simpler to grasp by focusing on the ASR. It is intriguing that males stop caring for offspring, and increase their participation in the more dangerous activity of competing for mates, yet this is readily explained by the femalebiased ASR. A male gains less than his mate by caring for offspring when, in the population as a whole, he is a member of the rarer sex and must therefore enjoy a higher reproductive rate than she does (the Fisher condition).

At generation 20 000, we assume an environmental reversal so that caring for offspring is now three times as dangerous as competing for mates. The immediate effect of this is a sudden spike towards a heavily male-biased OSR and ASR because males are not participating in the risky activity of caring for offspring. This male bias is, however, transient because there is immediately selection for increased male parental care. Although competing for mates is now a relatively safe activity, it is a less productive route to increase fitness because there are so many males competing for each female. As males evolve to spend more time caring this diminishes the newly created bias in the ASR because male mortality increases. The OSR also declines because males spend less time competing for mates. In contrast to the first 10000 generations, the last 10000 involve near egalitarian sex roles $(40 \%$ of care is by males, Fig. 5$)$.

\subsubsection{Do mammalian and avian care patterns simply reflect a difference in the ASR?}

When mate competition is the more dangerous activity, Fig. 5 shows evolution of a female-biased ASR (because of high male mortality), a male-biased OSR, and femaleonly care. This outcome is typical of most mammals. In contrast, if caring is costly the outcome is biparental care with females providing more care, and a male-biased OSR and ASR. This outcome is typical of that reported in many birds (Liker \& Székely, 2005; Donald, 2007). Although there is only a single phylogenetic contrast here, it is tempting to infer that the sex roles seen in birds and mammals are the result of taxon-specific differences 
in the relative mortality costs of caring and competing. Of course, these sex role differences might be the result of simple constraint (e.g. that the evolution of lactation is difficult for male mammals). More phylogenetic contrasts are needed. Encouragingly, however, bird species where males do not care also display a trend towards more mammal-like ASRs (Donald, 2007). Empiricists might like to collect data to test whether the ASR differences between clades with male-biased and female-biased care in other taxa differ in the same way.

Sex role divergence is hampered by a male-biased ASR because deserting males cannot all have a high per capita reproductive rate when they are the more common sex. The change in the course of evolution in Fig. 5 is entirely explained by the effect of changes in mortality for different activities on the OSR and ASR. All other parameters were kept constant. We did not assume that one sex was a priori rare or that the mortality rate for a given activity differed between the sexes. Caring was, for example, equally risky for both sexes. If other parameters evolve this could produce even more dramatic results. For example, if $\tilde{k}$ increases as sex roles diverge this would further amplify the difference between the OSR and the ASR in the 'mammalian' case (generations $10000 \ldots 20000)$.

\subsection{The fifth key conclusion: the ASR influences sex role divergence}

Based on Figs 4 and 5, we can add a fifth, and final, result.

Result 5: The ASR affects sex role evolution. The Fisher condition creates frequency-dependent selection that shifts parental investment until the equilibrium OSR is reached. Its value will depend on other parameters (e.g. sexual selection, multiple mating) but the relative parental investment by each sex that is necessary to produce a given OSR depends on the ASR. Therefore, any process that biases the ASR affects the evolution of sex roles. This does not simply depend on the ASR shifting the OSR from being male-biased to femalebiased. The more common sex is, all else being equal, under selection to avoid competition and provide more care instead. So, whenever sex role evolution biases the ASR because mortality costs differ between life history stages, sex roles will diverge less markedly if deserters become the more common sex in the ASR, and more markedly if they become the rarer sex.

\section{Conclusions}

Explaining sex role evolution is relatively easy if one assumes that only some traits and population level phenomena evolve (for an insightful discussion see McNamara et al., 2000). In reality, however, several highly influential factors must interact strongly (Houston et al., 2005). The coevolution of interlocked parameters makes it challenging to predict the outcome of a given scenario using verbal models (Kokko \& López-Sepulcre, 2007). The problem is exacerbated for sex roles given such a strong prior expectation of what the outcome should be (for a critique of 'conventional wisdom' see Levitt \& Dubner, 2005). There is a tendency to gloss over certain logical steps because the conclusion if foreshadowed (i.e. females care and males compete). In this review we have emphasized why certain widespread and deeply appealing arguments are flawed.

The belief that sex roles have an inherent tendency to diverge because anisogamy by itself produces conventional sex roles is demonstrably false. Formal mathematical models make it clear that mating is more difficult for the sex in the majority in the OSR (i.e. usually the sex with the higher PRR). Given the choice to care or compete there is relentless selection for egalitarian parental investment due to a fundamental property of populations (the Fisher condition). Sex roles only diverge if factors additional to (but possibly enhanced by) anisogamy are in play. On reflection this should be selfevident - if sex role divergence was an inevitable consequence of anisogamy, then sex-role reversal, biparental or male-only care, or mutual mate choice should not be possible.

We identified five key parameters to produce a general model that can help explain sex role evolution in most taxa. These are: the care needs of offspring, sexual selection, multiple mating or communal egg-laying and their influence on expected parentage, the OSR and the ASR. Of course, our model is only a starting point. We end this review by outlining some possible lines of future research.

\subsection{Sexual conflict: non-additive care and rapid adjust in response to a partner's effort}

We assumed that parental investment has an additive beneficial effect on offspring fitness, which generates clear sexual conflict over who cares. Non-additive benefits could, however, radically alter the outcome in specific cases. For example, synergistic benefits of biparental care (e.g. one parent forages while the other guards) will obviously reduce conflict and make biparental care more likely to evolve (Kokko \& Johnstone, 2002). Similarly, we ignored the possibility that parents can reduce offspring fitness (e.g. by filial cannibalism, which is common in fish; for a review see Klug $\&$ Bonsall, 2007). For simplicity we also ignored the complication that some breeding attempts will fail completely if one or more parents die while caring because it seems unlikely to bias the direction of sex role evolution. Similar issues arise if parents compensate for the loss of a carer (Royle et al., 2002; Houston et al., 2005) or use more complicated rules to adjust their level of care. As with earlier 'sealed bid' models of parental care (e.g. Winkler, 1987), our model assumes that parents do not monitor each 
others' efforts, thus changes in parental efforts only occur over evolutionary time. Removing this simplification is unlikely to bias caring towards one sex, but its effect on evolutionary trajectories could be tested.

\subsection{What happens when the strength of sexual selection evolves?}

Sexual selection ( $k$ and $\tilde{k}$ ) is a fixed parameter in our model. We decided not to make it a function of the OSR, in part, because of the wide range of plausible relationships. Allowing $k$ and $\tilde{k}$ to evolve is, however, a logical extension of our model. Apart from direct effects of the OSR on $k$ and $\tilde{k}$, there are biologically relevant withinindividual trade-offs that are worth investigating. For example, Kokko \& Wong (2007) recently investigated how sexual differences in mate-searching effort are related to mating success, mortality costs of searching, Bateman gradients and the OSR. So far, surprisingly few models explicitly consider the trade-off between mating effort and parental effort (e.g. Dawson, 1996). Such trade-offs can be profitably explored if they are directly related to the Bateman gradients for each sex.

Incorporating the evolution of mate choice into our model is another obvious future challenge. Choosiness, which elevates $k$ or $\tilde{k}$, partly depends on how often individuals encounter potential mates (Kokko \& Mappes, 2005). Again, this should lead to coevolution of $\tilde{k}, k$ and the OSR. It is important to remember that both sexes can be simultaneously choosy and competitive (Owens $\&$ Thompson, 1994; Johnstone et al., 1996). Because mate choice can evolve with great rapidity due to genetic correlations between traits (Mead \& Arnold, 2004), it is ultimately desirable that we build models that explicitly allow care, competitiveness and choosiness to evolve in both sexes, rather than restrict the evolution of some traits to one sex and the remainder to the other (e.g. Mylius, 1999; Seki et al., 2007).

\subsection{What if there is short-term adjustment of care to future mating prospects?}

Incorporating differential allocation and quality-dependent mate choice might explain why sex roles diverge more in some species than others. First, in our model behavioural plasticity in care is absent. Selection favours desertion by individuals with high future mating success, but we did not model reaction norms that would allow mated individuals to vary their care duration according to individual-specific expected future mating rates (higher or lower than that of an average mated individual). A mated individual (say a male), if able to correctly predict his future mating rate, might benefit by providing less parental care if the rate was above average for a mated male and more care if it was below average (Møller \& Thornhill, 1998) (Fig. 1). This could, in turn, select for females that care more for the offspring of more successful males and less for those of less successful males ('differential allocation', Sheldon, 2000). The same predictions apply for females.

Second, mate choice is sometimes for individuals that provide direct benefits by offering more parental care. At other times it is for individuals who provide genetic benefits, even at the cost of a reduction in parental care. The extent to which direct and genetic benefits are sought can be adaptively adjusted based on an individual's own quality. Male mate choice, for example, may evolve more easily if males can perceive and respond to their own competitive ability (Fawcett \& Johnstone, 2003; Härdling \& Kokko, 2005; Bel-Venner et al., 2008) than if they cannot (Servedio, 2007), and the different scenarios can involve a range of assortative mating patterns that might feed back and impact parental investment evolution.

\subsection{Lost paternity affects sex role evolution even if there is no behavioural plasticity}

Few studies directly emphasize how important females mating multiply and sperm competition are for the evolution of divergent sex roles. In contrast, many studies have asked how imperfect cues of paternity in socially monogamous species affect male care. Unfortunately, until quite recently, empiricists have often failed to distinguish between models designed to predict within vs. across species relationships, citing the same model in both contexts (e.g. model of Xia, 1992).

Debate about how uncertainty of paternity affects male parental care, primarily in the context of extra-pair paternity in birds, has focused on what factors favour the requisite male behavioural plasticity. That is, can males identify and respond to cues associated with loss of paternity? If so, when should they defer investment and save it for future broods (for a review see Sheldon, 2002)? The latter is a question about the response to variation around a mean. In contrast, models designed to address the long-term evolution of care consistently show that a decline in mean paternity per mating event selects for reduced male care (Yamamura \& Tsuji, 1993; Queller, 1997; Kokko, 1999; this study). This relationship should be observed at the cross-species (and possibly population) level, and it could explain much variation in paternal investment over evolutionary time scales. Empirical data from taxa other than birds are, however, in short supply.

\subsection{Multiple mating and sexual selection: an alluring trade-off}

Sexual selection, which leads to a subset of individuals mating, might well coevolve with multiple mating, which results in only a subset of offspring being genetically related to a putative parent. In our model 
these two 'subsets' of individuals have a near-identical influence on sex role evolution (e.g. Fig. 4). Of course, to identify their relative importance in nature we have to estimate actual parameter values. We have repeatedly noted how important it is to specify all plausible alternative routes to fitness when considering the evolved response to a shift in any single parameter. This is especially relevant in the case of selection imposed by female promiscuity and female choice. For example, a typical evolutionary response of males to female promiscuity is increased investment in the testes (e.g. Stockley et al., 1997) or sperm (e.g. Balshine et al., 2001). But will this, in turn, trade off with mating success? For example, in dungbeetles testis size trades off with weapon size (Simmons $\&$ Emlen, 2006). Coevolution can even produce a vicious circle where females try to avoid becoming sperm limited by mating multiply, and males become even more sperm-depleted as they produce ever larger ejaculates to decrease the potential loss of paternity as sperm competition intensifies (Charlat et al., 2007).

\subsection{OSR and ASR}

We think that one of the most useful results of our model is to help clarify the relationship between the OSR and ASR. This might provide opportunities for both theoreticians and empiricists. Models of the evolution of parental care tend to speak of 'sex ratios' in a rather general sense and rarely make explicit reference to the OSR (e.g. Grafen \& Sibly, 1978; Yamamura \& Tsuji, 1993; Queller, 1997; McNamara et al., 2000). In contrast, sexual selection models usually place great emphasis on the OSR (e.g. Clutton-Brock \& Parker, 1992; Parker \& Simmons, 1996; Kokko \& Monaghan, 2001).

We hope that our review of both sets of literature and consideration of both sex ratios in our model will encourage researchers to pay greater attention to the insights gained by distinguishing between the two sex ratios. When the ASR was allowed to coevolve with parental investment patterns, our model produced several results that, at least for us, were strongly counterintuitive from the perspective of the immediate effect of increasing the risk associated with an activity. However, these were readily explicable once the effects of mortalities on population-wide sex ratios were considered. Exploration of these causal routes to less or more likely divergence in sex roles deserves greater consideration.

It is encouraging that, despite theoreticians' disinterest, empiricists have quietly been gathering evidence about the importance of the population-wide ASR for sex role evolution (e.g. Jiggins et al., 2000; Forsgren et al., 2004; Donald, 2007; Heinsohn et al., 2007; Kvarnemo et al., 2007; Sogabe \& Yanagisawa, 2007; Foellmer, 2008; Pomfret \& Knell, 2008). More generally, empiricists have been attuned to the effect of variation in mating oppor- tunities, although the proxy measures they use to calculate this vary widely (compare Owens, 2002 with Olson et al., 2008). On the downside, there has been an unwarranted tendency for empiricists to relate their results to the OSR when they are, in effect, manipulating the ASR in small experimental populations (e.g. Alonso-Pimentel \& Papaj, 1999; Jirotkul, 1999; Klemme et al., 2007).

\subsection{When in the jungle, sharpen up your panga}

It is difficult to make sense of a tangle of causal relationships when various factors not only interact to influence the main outcome, but simultaneously influence each other. The challenge is to identify any clear predictions that emerge. One prediction we have made in this review is that when the relative risk of caring is higher than that of competing this diminishes the evolution of sex role divergence (all else being equal). Nevertheless, highlighting the importance of one factor at the expense of others can mislead (see Simmons $\delta$ Kvarnemo, 2006 on Kokko \& Monaghan, 2001; see also Fitze $\&$ Le Galliard, 2008). The challenge for empiricists is to avoid conflating the usefulness of any 'take home message' with the necessity of considering all the parameters in our equations (e.g. eqns $3 a$ and $3 \mathrm{~b})$. They should then use their hard-won insights into their own study systems to identify those parameters which are most or least likely to differ across environments or between the sexes. Testing whether this is indeed the case, and if so whether sex roles have evolved in the predicted directions, should help to identify the most important factors where it really matters - in nature.

\section{Acknowledgments}

We have discussed these ideas with numerous people, receiving much valuable advice. Lutz Fromhage, Jaco Greeff, Katja Heubel, Jan Heuschele, Hope Klug, Niclas Kolm, Lotta Kvarnemo, Jussi Lehtonen, Kai Lindström, Andrés López-Sepulcre, John McNamara, Daniel Rankin, Anna Rotkirch, Leigh Simmons, Wouter Vahl, Marlene Zuk and the entire LEED::ANTZZ discussion group deserve particular mention. We thank Bob Trivers and Staffan Ulfstrand for encouragement, two anonymous reviewers for further guidance, and Jean Drayton and Pat Backwell for assistance. We also thank Kristina Brahe for inspiration and The Gods. Funding was provided by the Academy of Finland and the Australian Research Council.

\section{References}

Ackerman, J.T. \& Eadie, J.M. 2003. Current versus future reproduction: an experimental test of parental investment decisions using nest desertion by mallards (Anas platyrhynchos). Behav. Ecol. Sociobiol. 54: 264-273. 
Ah-King, M., Kvarnemo, C. \& Tullberg, B.S. 2005. The influence of territoriality and mating system on the evolution of male care: a phylogenetic study on fish. J. Evol. Biol. 18: $371-382$.

Ahnesjö, I., Kvarnemo, C. \& Merilaita, S. 2001. Using potential reproductive rates to predict mating competition among individuals qualified to mate. Behav. Ecol. 12: 397-401.

Alcock, J. 1998. Animal Behavior: An Evolutionary Approach, 6th edn. Sinaueur Associates, Sunderland.

Alonso-Pimentel, H. \& Papaj, D.R. 1999. Resource presence and operational sex ratio as determinants of copulation duration in the fly Rhagoletis juglandis. Anim. Behav. 5: 1063-1069.

Arnold, S.J. \& Duvall, D. 1994. Animal mating systems: a synthesis based on selection theory. Am. Nat. 143: 317-348.

Ball, M.A. \& Parker, G.A. 2007. Sperm competition games: the risk model can generate higher sperm allocation to virgin females. J. Evol. Biol. 20: 767-779.

Balshine, S., Leach, B.J., Neat, F., Werner, N.Y. \& Montgomerie, R. 2001. Sperm size of African cichlids in relation to sperm competition. Behav. Ecol. 12: 726-731.

Balshine-Earn, S. 1995. The costs of parental care in Galilee St Peters fish, Sarotherodon galilaeus. Anim. Behav. 50: 1-7.

Balshine-Earn, S. \& Earn, D.J.D. 1998. On the evolutionary pathway of parental care in mouth-brooding cichlid fish. Proc. R. Soc. Lond., B, Biol. Sci. 265: 2217-2222.

Bateman, A.J. 1948. Intra-sexual selection in Drosophila. Heredity 2: $349-368$.

Baylis, J.R. 1981. The evolution of parental care in fishes, with reference to Darwin's rule of male sexual selection. Environ. Biol. Fishes 6: 223-251.

Beck, C.W. 1998. Mode of fertilization and parental care in anurans. Anim. Behav. 55: 439-449.

Bel-Venner, M.C., Dray, S., Allainé, D., Menu, F. \& Venner, S. 2008. Unexpected male choosiness for mates in a spider. Proc. R. Soc. Lond., B, Biol. Sci. 275: 77-82.

Benito, M.M. \& González-Solís, J. 2007. Sex ratio, sex-specific chick mortality and sexual size dimorphism in birds. J. Evol. Biol. 20: 1522-1530.

Berglund, A., Bisazza, A. \& Pilastro, A. 1996. Armaments and ornaments: an evolutionary explanation of traits of dual utility. Biol. J. Linn. Soc. 58: 385-399.

Blanckenhorn, W.U. 2005. Behavioral causes and consequences of sexual size dimorphism. Ethology 111: 977-1016.

Breitwisch, R. 1989. Mortality patterns, sex ratios, and parental investment in monogamous birds. In: Current Ornithology, Vol. 6 (D.M. Power, ed.), pp. 1-50. Plenum Press, New York.

Bro-Jørgensen, J. 2007. The intensity of sexual selection predicts weapon size in male bovids. Evolution 61: 1316-1326.

Bulmer, M.G. \& Parker, G.A. 2002. The evolution of anisogamy: a game-theoretic approach. Proc. R. Soc. Lond., B, Biol. Sci. 269: 2381-2388.

Byrne, P.G., Roberts, J.D. \& Simmons, L.W. 2002. Sperm competition selects for increased testes mass in Australian frogs. J. Evol. Biol. 15: 347-355.

Charlat, S., Reuter, M., Dyson, E.A., Hornett, E.A., Duplouy, A., Davies, N., Roderick, G.K., Wedell, N. \& Hurst, G.D.D. 2007. Male-killing bacteria trigger a cycle of increasing male fatigue and female promiscuity. Curr. Biol. 17: 273-277.

Clutton-Brock, T.H. 1991. The Evolution of Parental Care. Princeton University Press, Princeton.

Clutton-Brock, T.H. 2007. Sexual selection in males and females. Science 318: 1882-1885.
Clutton-Brock, T.H. \& Isvaran, K. 2007. Sex differences in ageing in natural populations of vertebrates. Proc. R. Soc. Lond., B, Biol. Sci. 274: 3097-3104.

Clutton-Brock, T.H. \& Parker, G.A. 1992. Potential reproductive rates and the operation of sexual selection. Q. Rev. Biol. 67: $437-456$.

Clutton-Brock, T.H. \& Vincent, A.C.J. 1991. Sexual selection and the potential reproductive rates of males and females. Nature 351: $58-60$.

Clutton-Brock, T.H., Albon, S.D. \& Guinness, F.E. 1985. Parental investment and sex differences in juvenile mortality in birds and mammals. Nature 313: 131-133.

Cockburn, A. 2006. Prevalence of different modes of parental care in birds. Proc. R. Soc. Lond., B, Biol. Sci. 273: 1375-1383.

Coleman, R.M. \& Gross, M.R. 1991. Parental investment theory - the role of past investment. Trends Ecol. Evol. 6: 404-406.

Coleman, R.M., Gross, M.R. \& Sargent, R.C. 1985. Parental investment decision rules: a test in bluegill sunfish. Behav. Ecol. Sociobiol. 18: 59-66.

Dawkins, R. 1989. The Selfish Gene, 2nd edn. Oxford University Press, Oxford.

Dawkins, R. \& Carlisle, T.R. 1976. Parental investment, mate desertion and a fallacy. Nature 262: 131-133.

Dawson, K.J. 1996. Evolutionary consequences of a trade-off between parental effort and mating effort. J. Theor. Biol. 183: 139-158.

Donald, P.F. 2007. Adult sex ratios in wild bird populations. Ibis 149: 671-692.

Duckworth, R.A., Badyaev, A.V. \& Parlow, A.F. 2003. Elaborately ornamented males avoid costly parental care in the house finch (Carpodacus mexicanus): a proximate perspective. Behav. Ecol. Sociobiol. 55: 176-183.

Düsing, C. 1884. Die Regulierung des Geschlechtsverhältnisses bei der Vermehrung der Menschen, Tiere und Pflanzen. Fischer, Jean.

Edwards, A.W.F. 2000. Carl Düsing (1884) on the regulation of the sex-ratio. Theor. Popul. Biol. 58: 255-257.

Emlen, S.T. \& Oring, L.W. 1977. Ecology, sexual selection, and the evolution of mating systems. Science 197: 215-223.

Fairbairn, D.J., Blanckenhorn, W.U. \& Székely, T. 2007. Sex, Size and Gender Roles: Evolutionary Studies of Sexual Size Dimorphism. Oxford University Press, Oxford.

Fawcett, T.W. \& Johnstone, R.A. 2003. Mate choice in the face of costly competition. Behav. Ecol. 14: 771-779.

Field, J. \& Brace, S. 2004. Pre-social benefits of extended parental care. Nature 428: 650-652.

Fisher, R.A. 1930. The Genetical Theory of Natural Selection. Oxford University Press, Oxford.

Fitze, P.S. \& Le Galliard, F. 2008. Operational sex ratio, sexual conflict and the intensity of sexual selection. Ecol. Lett. 11: 432-439.

Foellmer, M.W. 2008. Broken genitals function as mating plugs and affect sex ratios in the orb-web spider argiope aurantia. Evol. Ecol. Res. 10: 449-462.

Forsgren, E., Amundsen, T., Borg, Å.A. \& Bjelvenmark, J. 2004. Unusually dynamic sex roles in a fish. Nature 429: $551-554$

Frank, S.A. 1990. Sex allocation theory for birds and mammals. Annu. Rev. Ecol. Syst. 21: 13-55.

Fromhage, L., Elgar, M.A. \& Schneider, J.M. 2005. Faithful without care: the evolution of monogyny. Evolution 59: 14001405 . 
Fromhage, L., McNamara, J.M. \& Houston, A.I. 2007. Stability and value of male care for offspring: is it worth only half the trouble? Biol. Lett. 3: 234-236.

Fromhage, L., McNamara, J.M. \& Houston, A.I. 2008. A model for the evolutionary maintenance of monogyny in spiders. J. Theor. Biol. 250: 524-531.

García-González, F., Nunez, Y., Ponz, F., Roldan, E.R.S. \& Gomendio, M. 2003. Sperm competition mechanisms, confidence of paternity, and the evolution of paternal care in the golden egg bug (Phyllomorpha laciniata). Evolution 57: 10781088.

Goodwin, N.B., Balshine-Earn, S. \& Reynolds, J.D. 1998. Evolutionary transitions in parental care in cichlid fish. Proc. R. Soc. Lond., B, Biol. Sci. 265: 2265-2272.

Goodwin, N.B., Dulvy, N.K. \& Reynolds, J.D. 2002. Life-history correlates of the evolution of live bearing in fishes. Philos. Trans. R. Soc. Lond., B, Biol. Sci. 357: 259-267.

Grafen, A. \& Sibly, R. 1978. A model of mate desertion. Anim. Behav. 26: 645-652.

Griffith, S.C., Owens, I.P.F. \& Thuman, K.A. 2002. Extra pair paternity in birds: a review of interspecific variation and adaptive function. Mol. Ecol. 11: 2195-2212.

Gross, M.R. 1996. Alternative reproductive strategies and tactics: diversity within sexes. Trends Ecol. Evol. 11: A92A98.

Gross, M.R. 2005. The evolution of parental care. Q. Rev. Biol. 80: $37-45$.

Gubernick, D.J. \& Teferi, T. 2000. Adaptive significance of male parental care in a monogamous mammal. Proc. R. Soc. Lond., B, Biol. Sci. 267: 147-150.

Gwynne, D.T. 1981. Sexual difference theory: mormon crickets show role reversal in mate choice. Science 213: 779-780.

Gwynne, D.T. 1990. Testing parental investment and the control of sexual selection in katydids: the operational sex-ratio. Am. Nat. 136: 474-484.

Gwynne, D.T. \& Simmons, L.W. 1990. Experimental reversal of courtship roles in an insect. Nature 346: 172-174.

Hale, R.E. \& St Mary, C.M. 2007. Nest tending increases reproductive success, sometimes: environmental effects of paternal care and mate choice in flagfish. Anim. Behav. 74: $577-588$

Harada, Y. \& Iwasa, Y. 1996. Female mate preference to receive maximum paternal care: a two-step game. Am. Nat. 147: 9961027.

Härdling, R. \& Kokko, H. 2005. The evolution of prudent choice. Evol. Ecol. Res. 7: 697-715.

Härdling, R., Kokko, H. \& Arnold, K.E. 2003. Dynamics of the caring family. Am. Nat. 161: 395-412.

Heinsohn, R., Ebert, D., Legge, S. \& Peakall, R. 2007. Genetic evidence for cooperative polyandry in reverse dichromatic Eclectus parrots. Anim. Behav. 74: 1047-1054.

Hoelzer, G.A. 1989. The good parent process of sexual selection. Anim. Behav. 38: 1067-1078.

Houston, A.I. \& McNamara, J.M. 2002. A self-consistent approach to paternity and parental effort. Phil. Trans. R. Soc. Lond. B 357: 351-362.

Houston, A.I. \& McNamara, J.M. 2005. John Maynard Smith and the importance of consistency in evolutionary game theory. Biol. Philos. 20: 933-950.

Houston, A.I., Gasson, C. \& McNamara, J.M. 1997. Female choice of matings to maximize parental care. Proc. R. Soc. Lond., B, Biol. Sci. 264: 173-179.
Houston, A.I., Székely, T. \& McNamara, J.M. 2005. Conflict between parents over care. Trends Ecol. Evol. 20: 33-38.

Hunt, J. \& Simmons, L.W. 2002. Confidence of paternity and paternal care: covariation revealed through the experimental manipulation of the mating system in the beetle Onthophagus taurus. J. Evol. Biol. 15: 784-795.

Jennions, M.D. \& Petrie, M. 2000. Why do females mate multiply? Biol. Rev. 72: 21-64.

Jennions, M.D. \& Polakow, D.A. 2001. The effect of partial brood loss on male desertion in a cichlid fish: an experimental test. Behav. Ecol. 12: 84-92.

Jennions, M.D., Backwell, P.R.Y. \& Passmore, N.I. 1992. Breeding behavior of the African frog Chiromantis xerampelina: multiple spawning and polyandry. Anim. Behav. 44: 1091-1100.

Jiggins, F.M., Hurst, G.D.D. \& Majerus, M.E.N. 2000. Sex-ratiodistorting Wolbachia causes sex-role reversal in its butterfly host. Proc. R. Soc. Lond., B, Biol. Sci. 267: 69-73.

Jirotkul, M. 1999. Operational sex ratio influences female preference and male-male competition in guppies. Anim. Behav. 58: 287-294.

Johnstone, R.A., Reynolds, J.D. \& Deutsch, J.C. 1996. Mutual mate choice and sex differences in choosiness. Evolution 50: 1382-1391.

Keenleyside, M.H.A. 1983. Mate desertion in relation to adult sex-ratio in the biparental cichlid fish Herotilapia multispinosa. Anim. Behav. 31: 683-688.

Keenleyside, M.H.A. 1985a. Bigamy and mate choice in the biparental cichlid fish Cichlasoma nigrofasciatum. Behav. Ecol. Sociobiol. 17: 285-290.

Keenleyside, M.H.A. 1985b. Effects of altered adult sex ratio on the behavior of breeding Herotilapia multispinosa (Teleostei, Cichlidae). Neth. J. Zool. 35: 32-44.

Keenleyside, M.H.A. \& Mackereth, R.W. 1992. Effects of loss of male parent on brood survival in a biparental cichlid fish. Environ. Biol. Fishes 34: 207-212.

Klemme, I., Ylönen, H. \& Eccard, J.A. 2007. Reproductive success of male bank voles (Clethrionomys glareolus): the effect of operational sex ratio and body size. Behav. Ecol. Sociobiol. 61: 1911-1918.

Klug, H. \& Bonsall, M.B. 2007. When to care for, abandon, or eat your offspring: the evolution of parental care and filial cannibalism. Am. Nat. 170: 886-901.

Kokko, H. 1999. Cuckoldry and the stability of biparental care. Ecol. Lett. 2: 247-255.

Kokko, H. \& Jennions, M. 2003a. It takes two to tango. Trends Ecol. Evol. 18: 103-104.

Kokko, H. \& Jennions, M. 2003b. Response to McDowall: in defence of the caring male. Trends Ecol. Evol. 18: 611-612.

Kokko, H. \& Johnstone, R. A. 2002. Why is mutual mate choice not the norm? Operational sex ratios, sex roles, and the evolution of sexually dimorphic and monomorphic signalling Phil. Trans. R. Soc. Lond. B 357: 319-330.

Kokko, H. \& López-Sepulcre, A. 2007. The ecogenetic link between demography and evolution: can we bridge the gap between theory and data? Ecol. Lett. 10: 773-782.

Kokko, H. \& Mappes, J. 2005. Sexual selection when fertilization is not guaranteed. Evolution 59: 1876-1885.

Kokko, H. \& Monaghan, P. 2001. Predicting the direction of sexual selection. Ecol. Lett. 4: 159-165.

Kokko, H. \& Rankin, D.J. 2006. Lonely hearts or sex in the city? Density-dependent effects in mating systems Phil. Trans. R. Soc. Lond. B 361: 319-334. 
Kokko, H. \& Wong, B.B.M. 2007. What determines sex roles in mate searching? Evolution 61: 1162-1175.

Kokko, H., Jennions, M.D. \& Brooks, R. 2006. Unifying and testing models of sexual selection. Ann. Rev. Ecol. Evol. Syst. 37: 43-66.

Kvarnemo, C. 2006. Evolution and maintenance of male care: is increased paternity a neglected benefit of care? Behav. Ecol. 17: $144-148$.

Kvarnemo, C. \& Merilaita, S. 2006. Mating distribution and its temporal dynamics affect operational sex ratio: a simulation study. Biol. J. Linn. Soc. 89: 551-559.

Kvarnemo, C., Moore, G.I. \& Jones, A.G. 2007. Sexually selected females in the monogamous Western Australian seahorse. Proc. R. Soc. Lond., B, Biol. Sci. 274: 521-525.

Lavery, R.J. \& Keenleyside, M.H.A. 1990. Parental investment of a biparental cichlid fish, Cichlasoma nigrofasciatum, in relation to brood size and past investment. Anim. Behav. 40: 11281137.

Le Galliard, J.-F., Fitze, P.S., Ferrière, R. \& Clobert, J. 2005. Sex ratio bias, male aggression, and population collapse in lizards. Proc. Natl. Acad. Sci. U.S.A. 102: 18231-18236.

Lessells, C.M. 1998. A theoretical framework for sex-biased parental care. Anim. Behav. 56: 395-407.

Levitan, D.R. 1996. Effects of gamete traits on fertilization in the sea and the evolution of sexual dimorphism. Nature 382: 153155.

Levitan, D.R. 2002. Density-dependent selection on gamete traits in three congeneric sea urchins. Ecology 83: 464-479.

Levitan, D.R. \& Petersen, C. 1995. Sperm limitation in the sea. Trends Ecol. Evol. 10: 228-231.

Levitt, S. \& Dubner, S.J. 2005. Freakonomics: A Rogue Economist Explores the Hidden Side of Everything. Harper Collins, New York.

Liker, A. \& Székely, T. 2005. Mortality costs of sexual selection and parental care in natural populations of birds. Evolution $\mathbf{5 9}$ 890-897.

Lindenfors, P., Tullberg, B.S. \& Biuw, M. 2002. Phylogenetic analyses of sexual selection and sexual size dimorphism in pinnipeds. Behav. Ecol. Sociobiol. 52: 188-193.

Lorch, P.D. 2002. Understanding reversals in the relative strength of sexual selection on males and females: a role for sperm competition? Am. Nat. 159: 645-657.

MacColl, A.D.C. \& Hatchwell, B.J. 2003. Heritability of parental effort in a passerine bird. Evolution 57: 2191-2195.

Magrath, M.J.L. \& Komdeur, J. 2003. Is male care compromised by additional mating opportunity? Trends Ecol. Evol. 18: 424430.

Mank, J.E. \& Avise, J.C. 2006. Comparative phylogenetic analysis of male alternative reproductive tactics in ray-finned fishes. Evolution 60: 1311-1316.

Mank, J.E., Promislow, D.E.L. \& Avise, J.C. 2005. Phylogenetic perspectives in the evolution of parental care in ray-finned fishes. Evolution 59: 1570-1578.

Maynard Smith, J. 1977. Parental investment: a prospective analysis. Anim. Behav. 25: 1-9.

McNamara, J.M., Székely, T., Webb, J.N. \& Houston, A.I. 2000. A dynamic game-theoretic model of parental care. J. Theor. Biol. 205: 605-623.

Mead, L.S. \& Arnold, S.J. 2004. Quantitative genetic models of sexual selection. Trends Ecol. Evol. 19: 264-271.

Miller, J.A. 2007. Repeated evolution of male sacrifice behavior in spiders correlated with genital mutilation. Evolution 61: 1301-1315.
Mills, S.C. \& Reynolds, J.D. 2003. Operational sex ratio and alternative reproductive behaviours in the European bitterling, Rhodeus sericeus. Behav. Ecol. Sociobiol. 54: 98-104.

Mitchell, D.P., Dunn, P.O., Whittingham, L.A. \& FreemanGallant, C.R. 2007. Attractive males provide less parental care in two populations of the common yellowthroat. Anim. Behav. 73: $165-170$.

Møller, A.P. \& Birkhead, T.R. 1993. Certainty of paternity covaries with paternal care in birds. Behav. Ecol. Sociobiol. 33: 261-268.

Møller, A.P. \& Cuervo, J.J. 2000. The evolution of paternity and paternal care in birds. Behav. Ecol. 11: 472-485.

Møller, A.P. \& Jennions, M.D. 2001. How important are direct fitness benefits of sexual selection? Naturwissenschaften 88: 401-415.

Møller, A.P. \& Thornhill, R. 1998. Male parental care, differential parental investment by females and sexual selection. Anim. Behav. 55: 1507-1515.

Moore, S.L. \& Wilson, K. 2002. Parasites as a viability cost of sexual selection in natural populations of mammals. Science 297: 2015-2018.

Mylius, S.D. 1999. What pair formation can do to the battle of the sexes: towards more realistic game dynamics. J. Theor. Biol. 197: 469-485.

Neff, B.D. 2003. Decisions about parental care in response to perceived paternity. Nature 422: 716-719.

Olson, V.A., Liker, A., Freckleton, R.P. \& Székely, T. 2008. Parental conflict in birds: comparative analyses of offspring development, ecology and mating opportunities. Proc. R. Soc. Lond., B, Biol. Sci. 275: 301-307.

Owens, I.P.F. 2002. Male-only care and classical polyandry in birds: phylogeny, ecology and sex differences in remating opportunities. Phil. Trans. R. Soc. Lond. B 357: 283-293.

Owens, I.P.F. \& Thompson, D.B.A. 1994. Sex differences, sex ratios and sex roles. Proc. R. Soc. Lond., B, Biol. Sci. 258: 93-99.

Parker, G.A. 1990. Sperm competition games: raffles and roles. Proc. R. Soc. Lond., B, Biol. Sci. 242: 120-126.

Parker, G.A. \& Simmons, L.W. 1996. Parental investment and the control of sexual selection: predicting the direction of sexual competition. Proc. R. Soc. Lond., B, Biol. Sci. 263: 315-321.

Pomfret, J.C. \& Knell, R.J. 2008. Crowding, sex ratio and horn evolution in a South African beetle community. Proc. R. Soc. Lond., B, Biol. Sci. 275: 315-321.

Promislow, D.E.L. 1992. Costs of sexual selection in natural populations of mammals. Proc. R. Soc. Lond., B, Biol. Sci. 247: 203-210.

Promislow, D.E.L., Montgomerie, R. \& Martin, T.E. 1992. Mortality costs of sexual dimorphism in birds. Proc. R. Soc. Lond., B, Biol. Sci. 250: 143-150.

Queller, D.C. 1997. Why do females care more than males? Proc. R. Soc. Lond., B, Biol. Sci. 264: 1555-1557.

Reichard, M., Le Comber, S.C. \& Smith, C. 2007. Sneaking from a female perspective. Anim. Behav. 74: 679-688.

Reynolds, J.D. \& Jones, J.C. 1999. Female preference for preferred males is reversed under low oxygen conditions in the common goby (Pomatoschistus microps). Behav. Ecol. 10: $149-154$.

Reynolds, J.D., Goodwin, N.B. \& Freckleton, R.P. 2002. Evolutionary transitions in parental care and live bearing in vertebrates. Philos. Trans. R. Soc. Lond., B, Biol. Sci. 357: 269-281.

Richardson, P.R.K. 1987. Aardwolf mating system: overt cuckoldry in an apparently monogamous mammal. S. Afr. J. Sci. 83: 405-410. 
Ridley, A.R. \& Raihani, N.J. 2007. Variable postfledging care in a cooperative bird: causes and consequences. Behav. Ecol. 18: 994-1000.

Rios-Cardenas, O. \& Webster, A.S. 2005. Paternity and paternal effort in the pumpkinseed sunfish. Behav. Ecol. 16: 914-921.

Robertson, I.C. \& Roitberg, B.D. 1998. Duration of paternal care in pine engraver beetles: why do larger males care less? Behav. Ecol. Sociobiol. 43: 379-386.

Roff, D.A. 2002. Life History Evolution. Sinaeur Associates, Sunderland.

Royle, N.J., Hartley, I.R. \& Parker, G.A. 2002. Sexual conflict reduces offspring fitness in zebra finches. Nature 416: 733-736.

Sargent, R.C. \& Gross, M.R. 1985. Parental investment decision rules and the Concorde fallacy. Behav. Ecol. Sociobiol. 17: 43-45.

Sargent, R.C. \& Gross, M.R. 1993. William's principle: an explanation of parental care in teleost fishes. In: Behaviour of Teleost Fishes, 2nd edn (T.J. Pitcher, ed.), pp. 333-361. Chapman \& Hall, London.

Schwagmeyer, P.L., St Clair, R.C., Moodie, J.D., Lamey, T.C., Schnell, G.D. \& Moodie, M.N. 1999. Species differences in male parental care in birds: a reexamination of correlates with paternity. Auk 116: 487-503.

Segoli, M., Harari, A.R. \& Lubin, Y. 2006. Limited mating opportunities and male monogamy: a field study of white widow spiders, Latrodectus pallidus (Theridiidae). Anim. Behav. 72: 635-642.

Seki, M., Wakano, J.Y. \& Ihara, Y. 2007. A theoretical study on the evolution of male parental care and female multiple mating: effects of female mate choice and male care bias. J. Theor. Biol. 247: 281-296.

Servedio, M.R. 2007. Male versus female mate choice: sexual selection and the evolution of species recognition via reinforcement. Evolution 61: 2772-2789.

Sheldon, B.C. 2000. Differential allocation: tests, mechanisms and implications. Trends Ecol. Evol. 15: 397-402.

Sheldon, B.C. 2002. Relating paternity to paternal care. Philos. Trans. R. Soc. Lond., B, Biol. Sci. 357: 341-350.

Shuster, S.M. \& Wade, M.J. 2003. Mating Systems and Strategies. Princeton University Press, Princeton.

Simmons, L.W. \& Emlen, D.J. 2006. Evolutionary trade-off between weapons and testes. Proc. Natl. Acad. Sci. U.S.A. 103: 16346-16351.

Simmons, L.W. \& Kvarnemo, C. 2006. Costs of breeding and their effects on the direction of sexual selection. Proc. R. Soc. Lond., B, Biol. Sci. 273: 465-470.

Sogabe, A. \& Yanagisawa, Y. 2007. Sex-role reversal of a monogamous pipefish without higher potential reproductive rate in females. Proc. R. Soc. Lond., B, Biol. Sci. 274: 2959-2963.

Stockley, P., Gage, M.J.G., Parker, G.A. \& Møller, A.P. 1997. Sperm competition in fishes: the evolution of testis size and ejaculate characteristics. Am. Nat. 149: 933-954.

Summers, K., McKeon, C.S. \& Heying, H. 2006. The evolution of parental care and egg size: a comparative analysis in frogs. Proc. R. Soc. Lond., B, Biol. Sci. 273: 687-692.

Summers, K., McKeon, C.S., Heying, H., Hall, J. \& Patrick, W. 2007. Social and environmental influences on egg size evolution in frogs. J. Zool. 271: 225-232.

Taborsky, M. 2001. The evolution of bourgeois, parasitic, and cooperative reproductive behaviors in fishes. J. Hered. 92: 100110 .

Tallamy, D.W. 2000. Sexual selection and the evolution of exclusive paternal care in arthropods. Anim. Behav. 60: 559-567.
Thomas, G.H. \& Székely, T. 2005. Evolutionary pathways in shorebird breeding systems: sexual conflict, parental care, and chick development. Evolution 59: 2222-2230.

Tomkins, J.L. \& Hazel, W. 2007. The status of the conditional evolutionarily stable strategy. Trends Ecol. Evol. 22: 522-528.

Trivers, R. 1972. Parental investment and sexual selection. In: Sexual Selection and the Descent of Man 1871-1971 (B. Campbell, ed.), pp. 139-179. Aldine Press, Chicago.

Trivers, R. 1985. Social Evolution. Benjamin-Cummings, Menlo Park, CA.

Tschirren, B., Fitze, P.S. \& Richner, H. 2003. Sexual dimorphism in susceptibility to parasites and cell-mediated immunity in great tit nestlings. J. Anim. Ecol. 72: 839-845.

Uller, T., Pen, I., Wapstra, E., Beukeboom, L.W. \& Komdeur, J. 2007. The evolution of sex ratios and sex-determining systems. Trends Ecol. Evol. 22: 292-297.

Wade, M.J. \& Shuster, S.M. 2002. The evolution of parental care in the context of sexual selection: a critical reassessment of parental investment theory. Am. Nat. 160: 285-292.

Wakano, J.Y. \& Ihara, Y. 2005. Evolution of male parental care and female multiple mating: game-theoretical and two-locus diploid models. Am. Nat. 166: E32-E44.

Webb, J.N., Houston, A.I., McNamara, J.M. \& Székely, T. 1999. Multiple patterns of parental care. Anim. Behav. 58: 983-993.

Weckerly, F.W. 1998. Sexual-size dimorphism: Influence of mass and mating systems in the most dimorphic mammals. J. Mammal. 79: 33-52.

Westneat, D.F. \& Sherman, P.W. 1993. Parentage and the evolution of parental behavior. Behav. Ecol. 4: 66-77.

Wiklund, C., Kaitala, A. \& Wedell, N. 1998. Decoupling of reproductive rates and parental expenditure in a polyandrous butterfly. Behav. Ecol. 9: 20-25.

Williams, C.G. 1966. Adaptation and Natural Selection: A Critique of Some Current Thought. Princeton University Press, Princeton.

Winkler, D.W. 1987. A general model for parental care. Am. Nat. 130: 526-543.

Wright, J. 1998. Helping-at-the-nest and group size in the Arabian Babbler Turdoides squamiceps. J. Avian Biol. 29: 105-112.

Wright, H.W.Y. 2006. Paternal den attendance is the best predictor of offspring survival in the socially monogamous bat-eared fox. Anim. Behav. 71: 503-510.

Xia, X. 1992. Uncertainty of paternity can select against paternal care. Am. Nat. 139: 1126-1129.

Yamamura, N. \& Tsuji, N. 1993. Parental care as a game. J. Evol. Biol. 6: 103-127.

Zeh, D.W. \& Smith, R.L. 1985. Paternal investment by terrestrial arthropods. Am. Zool. 25: 785-805.

Received 21 December 2007; revised 12 March 2008; accepted 12 March 2008

\section{Appendix: Deriving mating rates and stable sex ratios}

To derive mating rates and stable sex ratios that satisfy the Fisher condition, we must consider population-wide averages rather than those of the subset of successful individuals. These calculations depend on how long the average individual spends, before dying, in the 'time out' or 'time in' states. The first step is to assume a mating rate and derive these durations (Part 1). We then use these 
values to work out the operational sex ratio (Part 2), which allows us to 'interlock' the parameters in a selfconsistent way (Part 3; Houston et al., 2005; Kokko \& López-Sepulcre, 2007). Only certain mating rates are internally consistent: female and male mating rates determine the OSR, while the OSR specifies the relationship between female and male mating rate, so these two relationships must be compatible.

\section{Part 1: Time spent in 'time in' or 'time out'}

We begin by calculating the durations. We first consider females. Females end a 'time in' period either when they die (rate $\mu_{\mathrm{I}}$ ) or mate (rate $a$ ). When both rates are constant, female 'time in' is exponentially distributed with the mean duration $1 /\left(\mu_{\mathrm{I}}+a\right)$ :

$$
\frac{\int_{t=0}^{\infty} t\left(\mu_{I}+a\right) \mathrm{e}^{-\left(\mu_{I}+a\right) t} \mathrm{~d} t}{\int_{t=0}^{\infty}\left(\mu_{I}+a\right) \mathrm{e}^{-\left(\mu_{I}+a\right) t} \mathrm{~d} t}=\frac{1}{\mu_{I}+a}
$$

We now need to know how many times she enters the 'time in' state. She does so once when she reaches adulthood, and then a certain number of times afterwards she returns to the mating pool and mates again. Doing this successfully requires a transition from 'time in' to 'time out', then from 'time out' to 'time in'. The probability that from 'time in' the next state is 'time out', rather than dead, is $a /\left(\mu_{\mathrm{I}}+a\right)$. The probability that from 'time out' the next state is 'time in' rather than dead is the probability that an individual in 'time out' is alive after time $T$, which is exp $\left(-\mu_{\mathrm{O}} T\right)$. Thus, the net probability of returning is $p=a \exp$ $\left(-\mu_{\mathrm{O}} T\right) /\left(\mu_{\mathrm{I}}+a\right)$. This means that the total number of occasions a female enters 'time in' is $1 /(1-p)$, and multiplying this with the average duration $1 /\left(\mu_{\mathrm{I}}+a\right)$ we get the expected total time in during a lifetime (denoted $\left.L_{\mathrm{I}}\right)$ :

$$
\mathrm{L}_{\mathrm{I}}=\frac{1}{a\left(1-\mathrm{e}^{-\mu_{\mathrm{O}} T}\right)+\mu_{\mathrm{I}}}
$$

To quantify the total time a female spends in 'time out', denoted by $L_{\mathrm{O}}$, we use the recursion equation:

$$
L_{\mathrm{O}}=\frac{a}{\mu_{\mathrm{I}}+a}\left[d T_{\text {final }}+(1-d)\left[T+L_{\mathrm{O}}\right]\right]
$$

where $T_{\text {final }}$ is the expected duration of a 'time out' that ends because the female dies before she re-enters the mating pool, $d$ is the probability that this occurs (given a 'time out' period had started) and $T$ is the duration of a completed time out (i.e. the normal parental care period). We have $d=1-\exp \left(-\mu_{\mathrm{O}} T\right)$, and

$$
T_{\text {final }}=\frac{\int_{t=0}^{T} t \mu_{\mathrm{O}} \mathrm{e}^{-\mu_{\mathrm{O}} t} \mathrm{~d} t}{\int_{t=0}^{T} \mu_{\mathrm{O}} \mathrm{e}^{-\mu_{\mathrm{O}} t} \mathrm{~d} t}=\frac{1-\mathrm{e}^{\mu_{\mathrm{O}} T}+\mu_{\mathrm{O}} T}{\mu_{\mathrm{O}}\left(1-\mathrm{e}^{\mu_{\mathrm{O}} T}\right)}
$$

Solving from the above and simplifying, we obtain $L_{\mathrm{O}}$ :

$$
\mathrm{L}_{\mathrm{O}}=\frac{a T-a d\left(T-T_{\text {final }}\right)}{a d+\mu_{\mathrm{I}}}=\frac{a\left(1+\mu_{\mathrm{O}} T-\mathrm{e}^{\mu_{\mathrm{O}} T}\left(1-\mu_{\mathrm{O}} T\right)\right)}{\left(\mu_{\mathrm{I}}+a\left(1-\mathrm{e}^{\mu_{\mathrm{O}} T}\right)\right) \mu_{\mathrm{O}}}
$$

The calculations for males proceed exactly analogously. Note that expression (A5) is valid for any mating rate $a$, but only some rates are consistent with the Fisher condition. The next step is to relate $a$ to the sex ratios (OSR and ASR).

\section{Part 2: Sex ratios}

We denote the primary sex ratio as $r$ (males : females). As we assume no sex bias in juvenile mortality, $r$ simultaneously refers to the sex ratio at maturation. The operational and adult sex ratios are then obtained on the basis of the total lifetime that an average male or a female spends in either 'time in' (OSR) or in whichever state (ASR):

$$
r_{\mathrm{O}}=r \frac{\tilde{L}_{\mathrm{I}}}{L_{\mathrm{I}}}, \quad r_{\mathrm{A}}=r \frac{\tilde{L}_{\mathrm{I}}+\tilde{L}_{\mathrm{O}}}{L_{\mathrm{I}}+L_{\mathrm{O}}}
$$

These are not yet very useful expressions because the $L_{\mathrm{I}}$ and $L_{\mathrm{O}}$ variables depend on the value of $a$ (eqns A2 and A5), but $a$ in turn depends on $r_{\mathrm{O}}$ (the Fisher condition). We must therefore work out these interdependencies to perform the necessary 'interlocking'.

We denote the mean number of males and females in a mating group by $\tilde{n}$ and $n$ respectively. The rate at which individuals enter groups depends on $r_{\mathrm{O}}$ (the Fisher condition), but individuals of a particular sex also enter mating groups more often if groups contain more individuals of that sex. When combined, these arguments dictate:

$$
\frac{a}{\tilde{a}}=\frac{n}{\tilde{n}} r_{\mathrm{O}}=r \frac{n \tilde{L}_{\mathrm{I}}}{\tilde{n} L_{\mathrm{I}}}
$$

We then specify a parameter $M$ such that: $a=M r_{\mathrm{O}}^{1 / 2} n$ and $\tilde{a}=M r_{\mathrm{O}}^{-1 / 2} \tilde{n}$. This allows us to alter the mate encounter rate in the population without violating the Fisher condition, because $a / \tilde{a}$ simplifies to eqn (A7) regardless of the value of $M$. We can then obtain the stable OSR by combining eqns (A2), (A5) and (A7):

$$
\frac{n}{\tilde{n}} r_{\mathrm{O}}=r \frac{n \tilde{L}_{\mathrm{I}}}{\tilde{n} L_{\mathrm{I}}}=r \frac{n}{\tilde{n}} \frac{M r_{\mathrm{O}}^{1 / 2} n\left(1-\mathrm{e}^{-\mu_{\mathrm{O}} T}\right)+\mu_{\mathrm{I}}}{M r_{\mathrm{O}}^{-1 / 2} \tilde{n}\left(1-\mathrm{e}^{-\tilde{\mu}_{\mathrm{O}} \tilde{T}}\right)+\tilde{\mu}_{\mathrm{I}}}
$$

This leads to the unwieldy, yet useful, expression:

$$
r_{\mathrm{O}}=r \frac{\mu_{\mathrm{I}}}{\tilde{\mu}_{\mathrm{I}}}+\frac{M^{2} A}{2 \tilde{\mu}_{\mathrm{I}}^{2}}\left(A \pm \sqrt{A^{2}+\frac{4 \mu_{\mathrm{I}} \tilde{\mu}_{\mathrm{I}} r}{M^{2}}}\right),
$$

where $A=n r\left(1-\mathrm{e}^{-\mu_{\mathrm{O}} T}\right)-\tilde{n}\left(1-\mathrm{e}^{-\tilde{\mu}_{\mathrm{O}} \tilde{T}}\right)$.

As we now have closed form solutions for $r_{\mathrm{O}}, L_{\mathrm{O}}, L_{\mathrm{I}}$ and the male equivalents, the stable ASR is now obtained in closed form too:

$$
r_{\mathrm{A}}=r \frac{\tilde{L}_{\mathrm{I}}+\tilde{L}_{\mathrm{O}}}{L_{\mathrm{I}}+L_{\mathrm{O}}}
$$

